\title{
Front Matter: Volume 8012
}

, "Front Matter: Volume 8012," Proc. SPIE 8012, Infrared Technology and Applications XXXVII, 801201 (5 July 2011); doi: 10.1117/12.901326

SDIE Event: SPIE Defense, Security, and Sensing, 2011, Orlando, Florida, United SPIE. States 


\title{
PROCEEDINGS OF SPIE
}

\section{Infrared Technology and Applications XXXVII}

\author{
Bjørn F. Andresen \\ Gabor F. Fulop \\ Paul R. Norton \\ Editors
}

25-29 April 2011

Orlando, Florida, United States

Sponsored and Published by

SPIE 
The papers included in this volume were part of the technical conference cited on the cover and title page. Papers were selected and subject to review by the editors and conference program committee. Some conference presentations may not be available for publication. The papers published in these proceedings reflect the work and thoughts of the authors and are published herein as submitted. The publisher is not responsible for the validity of the information or for any outcomes resulting from reliance thereon.

Please use the following format to cite material from this book:

Author(s), "Title of Paper," in Infrared Technology and Applications XXXVII, edited by

Bjørn F. Andresen, Gabor F. Fulop, Paul R. Norton, Proceedings of SPIE Vol. 8012 (SPIE, Bellingham, WA, 2011) Article CID Number.

ISSN 0277-786X

ISBN 9780819485861

Published by

SPIE

P.O. Box 10, Bellingham, Washington 98227-0010 USA

Telephone +1 3606763290 (Pacific Time) · Fax +1 3606471445

SPIE.org

Copyright (C) 2011, Society of Photo-Optical Instrumentation Engineers

Copying of material in this book for internal or personal use, or for the internal or personal use of specific clients, beyond the fair use provisions granted by the U.S. Copyright Law is authorized by SPIE subject to payment of copying fees. The Transactional Reporting Service base fee for this volume is $\$ 18.00$ per article (or portion thereof), which should be paid directly to the Copyright Clearance Center (CCC), 222 Rosewood Drive, Danvers, MA 01923. Payment may also be made electronically through CCC Online at copyright.com. Other copying for republication, resale, advertising or promotion, or any form of systematic or multiple reproduction of any material in this book is prohibited except with permission in writing from the publisher. The CCC fee code is $0277-786 \mathrm{X} / 11 / \$ 18.00$.

Printed in the United States of America.

Publication of record for individual papers is online in the SPIE Digital Library.

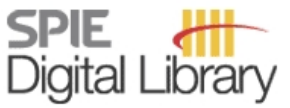

SPIEDigitalLibrary.org

Paper Numbering: Proceedings of SPIE follow an e-First publication model, with papers published first online and then in print and on CD-ROM. Papers are published as they are submitted and meet publication criteria. A unique, consistent, permanent citation identifier (CID) number is assigned to each article at the time of the first publication. Utilization of CIDs allows articles to be fully citable as soon as they are published online, and connects the same identifier to all online, print, and electronic versions of the publication. SPIE uses a six-digit CID article numbering system in which:

- The first four digits correspond to the SPIE volume number.

- The last two digits indicate publication order within the volume using a Base 36 numbering system employing both numerals and letters. These two-number sets start with $00,01,02,03,04$, $05,06,07,08,09,0 A, 0 B \ldots 0 Z$, followed by 10-1Z, 20-2Z, etc.

The CID number appears on each page of the manuscript. The complete citation is used on the first page, and an abbreviated version on subsequent pages. Numbers in the index correspond to the last two digits of the six-digit CID number. 


\title{
Contents
}

\section{Part One}

\author{
ixx Conference Committee \\ xxiii Introduction
}

\section{TARGET ACQUISITION WITH TODAY'S LEADING IMAGING TECHNOLOGIES}

801202 Sofradir latest developments for infrared space detectors [8012-01]

P. Chorier, A. Delannoy, SOFRADIR (France)

801203 First flights of a new airborne thermal infrared imaging spectrometer with high area coverage [8012-02]

J. L. Hall, R. H. Boucher, D. J. Gutierrez, S. J. Hansel, B. P. Kasper, E. R. Keim, N. M. Moreno, M. L. Polak, M. G. Sivjee, D. M. Tratt, D. W. Warren, The Aerospace Corp. (United States)

801204 SCD's uncooled detectors and video engines for a wide-range of applications [8012-03] A. Fraenkel, U. Mizrahi, L. Bikov, A. Giladi, N. Shiloah, S. Elkind, I. Kogan, S. Maayani, A. Amsterdam, I. Vaserman, O. Duman, Y. Hirsh, F. Schapiro, SCD Semiconductor Devices (Israel); A. Tuito, M. Ben-Ezra, Israel Ministry of Defense (Israel)

801205 The new megapixel thermal imager family [8012-04]

J. Fritze, M. Münzberg, Carl Zeiss Optronics GmbH (Germany)

$801207 \quad$ New applications with SWIR imager employing extended wavelengths [8012-07]

G. A. Tidhar, R. Segal, ELTA Systems LTD (Israel)

\section{THREAT IDENTIFICATION I}

801208 Blast investigation by fast multispectral radiometric analysis [8012-08]

A. D. Devir, Y. Bushlin, I. Mendelewicz, A. B. Lessin, M. Engel, IARD Sensing Solutions Ltd. (Israel)

801209 Open path FTIR detection of threat chemicals in air and on surfaces [8012-09]

J. R. Castro-Suarez, L. C. Pacheco-Londoño, W. Ortiz-Rivera, M. Vélez-Reyes, Univ. de Puerto Rico Mayagüez (United States); M. Diem, Northeastern Univ. (United States);

S. P. Hernandez-Rivera, Univ. de Puerto Rico Mayagüez (United States)

$80120 \mathrm{~A}$ Scene understanding and task optimisation using multimodal imaging sensors and context: a real-time implementation [8012-152]

B. Connor, Thales Optronics Ltd. (United Kingdom); J. Letham, N. Robertson, Heriot-Watt Univ. (United Kingdom); I. Carrie, Thales Optronics Ltd. (United Kingdom) 
8012 OB Simultaneous multispectral framing infrared camera using an embedded diffractive optical lenslet array [8012-11]

M. Hinnrichs, Pacific Advanced Technology, Inc. (United States)

$80120 \mathrm{C}$ The infrared-based early warning system for bird strike prevention at Frankfurt airport [8012-12]

M. Münzberg, A. Schilling, H. Schlemmer, H. Vogel, Carl Zeiss Optronics GmbH (Germany);

H. Cramer, J. Schlosshaver, Fusion Systems GmbH (Germany)

$80120 \mathrm{D}$ Time-varying phase diversity furbulence compensation [8012-13]

A. W. M. van Eekeren, K. Schutte, J. Dijk, P. B. W. Schwering, TNO Defence, Security and Safety (Netherlands)

\section{SMART IMAGE AND SIGNAL PROCESSING}

$80120 \mathrm{E}$ Focal plane generation of multi-resolution and multi-scale image representation for low-power vision applications (Invited Paper) [8012-14]

J. Fernández-Berni, R. Carmona-Galán, L. Carranza-González, CSIC-Univ. of Seville (Spain); A. Zarándy, Computer and Automation Research Institute (Hungary); Á. Rodríguez-Vázquez, CSIC-Univ. of Seville (Spain)

8012 OF Advanced multi-function infrared detector with on-chip processing (Invited Paper) [8012-15]

L. Langof, D. Nussinson, E. llan, S. Elkind, R. Dobromislin, I. Nevo, F. Khinich, M. Labilov,

Z. Calahorra, S. Vaserman, T. Markovitz, SCD Semiconductor Devices (Israel); O. Manela,

D. Elooz, Elbit Systems Electro-Optics El-Op Ltd. (Israel); A. Tuitto, D. Oster, Israeli Ministry of Defence (Israel)

801206 Analysis and simulation of CTIA-based pixel reset noise (Invited Paper) [8012-18]

D. A. Van Blerkom, Forza Silicon Corp. (United States)

$8012 \mathrm{OH}$ Calibration method for division of focal plane polarimeters in the optical and near-infrared regime (Invited Paper) [8012-19]

T. York, V. Gruev, Washington Univ. in St. Louis (United States)

801201 Hexagonal sampling in the infrared domain: an introduction to array set addressing (Invited Paper) [8012-157]

N. I. Rummelt, Air Force Research Lab. (United States); G. L. Barrows, Centeye, Inc. (United

States); M. A. Massie, Nova Sensors, Inc. (United States)

8012 0J A high fill-factor high-SNR CMOS image sensor for IR camera applications [8012-125]

V. Shenoy, D. McBride, S. Jung, H. Moon, The Univ. of Texas at Arlington (United States);

S.-Y. Cho, New Mexico State Univ. (United States)

8012 OK Design of multiple demeaning filters for small target detection in infrared imageries [8012-129]

W.-C. Choi, A. Heo, J.-H. Lee, D.-J. Park, Korea Advanced Institute of Science and

Technology (Korea, Republic of) 
$8012 \mathrm{OL}$ Image processing module for high-speed thermal camera with cooled detector [8012-130] G. Bieszczad, T. Sosnowski, H. Madura, M. Kastek, J. Bareła, Military Univ. of Technology (Poland)

8012 OM Design of ROIC based on switched capacitor TDI for MCT LWIR focal plane arrays [8012-131] H. Kayahan, M. Yazici, O. Ceylan, M. B. Baran, Y. Gurbuz, Sabanci Univ. (Turkey)

$80120 \mathrm{~N}$ Image interpolation methods evaluation for division of focal plane polarimeters [8012-133] S. Gao, V. Gruev, Washington Univ. in St. Louis (United States)

\section{QWIP AND QDIP}

801200 Thermo-electrically cooled shortwave infrared and longwave infrared dual-band quantum-dot photodetector [8012-20]

J. Vaillancourt, P. Vasinajindakaw, X. Lu, X. Qian, S. R. Vangala, W. D. Goodhue, Univ. of Massachusetts Lowell (United States)

8012 OP Design of broadband QWIPs [8012-21]

V. Guériaux, V. Trinité, A. Coulibaly, L. Dua, N. Brière de l'Isle, X. Marcadet, E. Costard, P. Bois, A. Nedelcu, Alcatel-Thales III-V Lab. (France)

$80120 Q$ Performance of the QWIP focal plane arrays for NASA's Landsat Data Continuity Mission [8012-22]

M. Jhabvala, NASA Goddard Space Flight Ctr. (United States); K. Choi, U.S. Army Research Lab. (United States); A. Waczynski, A. La, NASA Goddard Space Flight Ctr. (United States); M. Sundaram, QmagiQ, LLC (United States); E. Costard, Thales Research \& Technology (France); C. Jhabvala, E. Kan, D. Kahle, R. Foltz, NASA Goddard Space Flight Ctr. (United States); N. Boehm, M. Hickey, Global Science and Technology, Inc. (United States); J. Sun, U.S. Army Research Lab. (United States); T. Adachi, Catholic Univ. of America (United States); N. Costen, L. Hess, Munz Engineering Inc. (United States); H. Facoetti, Alcatel-Thales III-V Lab. (France); M. Montanaro, Sigma Space Corp. (United States)

8012 OR Electromagnetic modeling of QWIP FPA pixels [8012-23]

K. K. Choi, U.S. Army Research Lab. (United States); M. D. Jhabvala, NASA Goddard Space Flight Ctr. (United States); D. P. Forrai, L-3 Communications Cincinnati Electronics (United States); A. Waczynski, NASA Goddard Space Flight Ctr. (United States); J. Sun, U.S. Army Research Lab. (United States); R. Jones, L-3 Communications Cincinnati Electronics (United States)

\section{TYPE II SUPERLATTICE FPAS I}

8012 OS Update on III-V antimonide-based superlattice FPA development and material characterization (Invited Paper) [8012-24]

L. Zheng, Institute for Defense Analyses (United States); M. Tidrow, S. Bandara, L. Aitcheson, T. Shih, U.S. Army Night Vision \& Electronic Sensors Directorate (United States)

8012 OT Recent advances in high-performance antimonide-based superlattice FPAs (Invited Paper) [8012-25]

E. K. Huang, B.-M. Nguyen, S. Ramezani-Darvish, S. Abdollahi Pour, G. Chen, A. Haddadi, M.-A. Hoang, Northwestern Univ. (United States) 
8012 OU Current developments for type-Il superlattice imaging systems (Invited Paper) [8012-26] F. Rutz, R. Rehm, M. Walther, L. Kirste, M. Masur, A. Wörl, J. Schmitz, M. Wauro, J. Niemasz, Fraunhofer-Institute for Applied Solid State Physics (Germany); R. Scheibner, J. Ziegler, AIM INFRAROT-MODULE GmbH (Germany)

8012 OV Performance optimization of long-wave infrared detectors based on InAs/GaSb strained layer superlattices [8012-27]

E. Plis, B. Klein, N. Gautam, S. Myers, M. N. Kutty, M. Naydenkov, S. Krishna, Univ. of New Mexico (United States)

8012 OW Effects of carrier concentration and phonon energy on carrier lifetime in type-2 SLS and properties of InAs1-xSbx alloys [8012-28]

G. Belenky, G. Kipshidze, D. Donetsky, Stony Brook Univ. (United States); S. P. Svensson, W. L. Sarney, H. Hier, U.S. Army Research Lab. (United States); L. Shterengas, D. Wang, Y. Lin, Stony Brook Univ. (United States)

\section{TYPE II SUPERLATTICE FPAS II}

$80120 X \quad H i g h$ performance dual-band InAs/GaSb SLS detectors with nBn and pBp architectures [8012-29]

E. Plis, SK Infrared LLC (United States); N. Gautam, S. Myers, The Univ. of New Mexico (United States); S. S. Krishna, SK Infrared LLC (United States); E. P. Smith, S. Johnson, Raytheon Vision Systems (United States); S. Krishna, SK Infrared LLC (United States)

8012 OY Vertical transport in InAs/GaSb type-II strained layer superlattices for infrared focal plane array applications [8012-30]

G. A. Umana-Membreno, The Univ. of Western Australia (Australia); B. Klein, The Univ. of New Mexico (United States); H. Kala, J. Antoszewski, The Univ. of Western Australia (Australia);

G. Gautam, M. N. Kutty, E. Plis, The Univ. of New Mexico (United States); J. M. Dell, The Univ. of Western Australia (Australia); S. Krishna, The Univ. of New Mexico (United States);

L. Faraone, The Univ. of Western Australia (Australia)

$80120 Z$ Noise performance analysis of MWIR InAs/GaSb superlattice pin photodiodes [8012-31]

I. Ribet-Mohamed, K. Jaworowicz, D. Tayibi, ONERA (France); C. Cervera, R. Taalat,

J.-B. Rodriguez, P. Christol, Institut d'Electronique du Sud, CNRS, Univ. Montpellier 2 (France)

801210 Low-temperature noise measurements of an InAs/GaSb-based nBn MWIR detector [8012-32]

V. M. Cowan, C. P. Morath, Air Force Research Lab. (United States); S. Myers, N. Gautam,

S. Krishna, Univ. of New Mexico (United States)

801211 Scaling up antimonide wafer production: innovation and challenges for epitaxy ready GaSb and InSb substrates [8012-33]

M. J. Furlong, R. Martinez, S. Amirhaghi, D. Small, B. Smith, A. Mowbray, Wafer Technology Ltd. (United Kingdom)

801212 Fabrication and performance of InAs/GaSb-based superlattice LWIR detectors [8012-34] S. Terterian, H. Sharifi, P.-Y. Delaunay, B. Nosho, M. Roebuck, R. Rajavel, HRL Labs., LLC (United States) 
801213 Performances analysis of symmetrical and asymmetrical InAs/GaSb superlattice pin photodiode [8012-35]

C. Cervera, R. Taalat, P. Christol, J. B. Rodriguez, Institut d'Electronique du Sud, CNRS, Univ. Montpellier 2 (France); K. Jaworowicz, I. Ribet-Mohamed, ONERA (France); L. Konczewicz,

S. Contreras, Lab. Charles Coulomb, CNRS, Univ. Montpellier 2 (France)

801214 Superlattice barrier infrared detector development at the Jet Propulsion Laboratory (Invited Paper) [8012-36]

D. Z. Ting, A. Soibel, S. B. Rafol, J. Nguyen, L. Höglund, A. Khoshakhlagh, S. A. Keo, J. K. Liu, J. M. Mumolo, S. D. Gunapala, Jet Propulsion Lab. (United States)

801215 100mm diameter GaSb substrates with extended IR wavelength for advanced space-based applications [8012-124]

L. P. Allen, J. P. Flint, G. Meshew, G. Dallas, D. Bakken, Galaxy Compound Semiconductors, Inc. (United States); G. J. Brown, Air Force Research Lab. (United States); A. Khoshakhlagh, C. J. Hill, Jet Propulsion Lab. (United States)

NOTE: See also paper 801243 in the ORAL STANDBY/POSTERS session.

\section{EMERGING UNCOOLED TECHNOLOGIES}

801216 Toward 17um pitch heterogeneously integrated Si/SiGe quantum well bolometer focal plane arrays [8012-37]

P. Ericsson, Acreo AB (Sweden); A. C. Fischer, F. Forsberg, N. Roxhed, KTH-Royal Institute of Technology (Sweden); B. Samel, S. Savage, Acreo AB (Sweden); G. Stemme, KTH-Royal Institute of Technology (Sweden); S. Wissmar, O. Öberg, Acreo AB (Sweden); F. Niklaus, KTH-Royal Institute of Technology (Sweden)

801218 Infrared phased-array sensor [8012-39]

B. A. Slovick, CREOL, The College of Optics and Photonics, Univ. of Central Florida (United States); J. A. Bean, Georgia Tech Research Institute (United States); L. A. Florence,

G. D. Boreman, CREOL, The College of Optics and Photonics, Univ. of Central Florida (United States)

801219 High-speed uncooled MWIR hostile fire indication sensor [8012-40]

L. Zhang, F. P. Pantuso, G. Jin, A. Mazurenko, M. Erdtmann, S. Radhakrishnan, J. Salerno, Agiltron, Inc. (United States)

\section{UNCOOLED FPAS AND APPLICATIONS I}

80121 A Uncooled detector development at Raytheon [8012-41]

S. H. Black, T. Sessler, E. Gordon, R. Kraft, T. Kocian, M. Lamb, R. Williams, T. Yang, Raytheon Co. (United States)

8012 1B Development of terahertz focal plane arrays and handy camera [8012-42] N. Oda, M. Sano, K. Sonoda, H. Yoneyama, S. Kurashina, M. Miyoshi, T. Sasaki, NEC Corp. (Japan); I. Hosako, N. Sekine, National Institute of Information and Communications Technology (Japan); T. Sudou, S. Ohkubo, NEC Avio Infrared Technologies Co., Ltd. (Japan) 
$80121 \mathrm{~V} \quad$ VGA $17 \mu \mathrm{m}$ development for compact, low-power systems [8012-43]

A. Durand, J. L. Tissot, P. Robert, S. Cortial, C. Roman, M. Vilain, O. Legras, ULIS (France)

$80121 D \quad M o d u l a r$ uncooled video engines based on a DSP processor [8012-44]

F. Schapiro, Y. Milstain, A. Aharon, A. Neboshchik, Y. Ben-Simon, I. Kogan, I. Lerman,

U. Mizrahi, S. Maayani, A. Amsterdam, I. Vaserman, O. Duman, R. Gazit, SCD Semiconductor Devices (Israel)

$80121 \mathrm{E} \quad$ Development of new SOl diode structure for beyond $17 \mu \mathrm{m}$ pixel pitch SOl diode uncooled IRFPAs [8012-45]

D. Takamuro, T. Maegawa, T. Sugino, Y. Kosasayama, T. Ohnakado, H. Hata, M. Ueno, H. Fukumoto, K. Ishida, Mitsubishi Electric Corp. (Japan); H. Katayama, T. Imai, M. Ueno, Japan Aerospace Exploration Agency (Japan)

$80121 \mathrm{~F} \quad$ Improvements of a digital $25 \mu \mathrm{m}$ pixel-pitch uncooled amorphous silicon TEC-less VGA IRFPA with massively parallel Sigma-Delta-ADC readout [8012-46]

D. Weiler, M. Ruß, D. Würfel, R. Lerch, P. Yang, J. Baver, J. Heß, P. Kropelnicki, H. Vogt, Fraunhofer-Institut für Mikroelektronische Schaltungen und Systeme (Germany)

$80121 \mathrm{G}$ Scale down of p-n junction diodes of an uncooled IR-FPA for improvement of the sensitivity

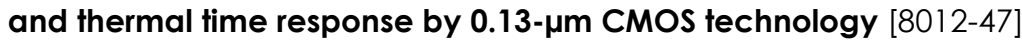

I. Fujiwara, K. Sasaki, K. Suzuki, H. Yagi, H. Kwon, H. Honda, K. Ishii, M. Ogata, M. Atsuta,

R. Ueno, M. Kobayashi, H. Funaki, Toshiba Corp. (Japan)

$80121 \mathrm{H} \quad 1024 \times 768$ XGA uncooled camera core achieves new levels of performance in a small package [8012-150]

C. J. Alicandro, R. W. DeMarco, Sofradir EC, Inc. (United States)

$801211 \quad$ Pixel level packaging for uncooled IRFPA [8012-49]

G. Dumont, W. Rabaud, X. Baillin, J. L. Pornin, L. Carle, V. Goudon, C. Vialle, M. Pellat, A. Arnaud, CEA-LETI-MINATEC (France)

\section{UNCOOLED FPAS AND APPLICATIONS II}

$80121 \mathrm{~K}$ Comparison of ion beam and magnetron sputtered vanadium oxide thin films for uncooled IR imaging [8012-51]

O. M. Cabarcos, J. Li, B. D. Gauntt, The Pennsylvania State Univ. (United States); S. Antrazi, 4Wave Inc. (United States); E. C. Dickey, North Carolina State Univ. (United States); D. L. Allara, M. W. Horn, The Pennsylvania State Univ. (United States)

$80121 \mathrm{~L}$ Performance improvement in amorphous silicon based uncooled microbolometers through pixel design and materials development [8012-52]

S. Ajmera, J. Brady, C. Hanson, T. Schimert, A. J. Syllaios, M. Taylor, L-3 Communications Electro-Optical Systems (United States)

$80121 \mathrm{M}$ Uncooled infrared detectors toward smaller pixel pitch with newly proposed pixel structure [8012-53]

S. Tohyama, T. Sasaki, T. Endoh, M. Sano, K. Katoh, S. Kurashina, M. Miyoshi, T. Yamazaki, NEC Corp. (Japan); M. Ueno, H. Katayama, T. Imai, Japan Aerospace Exploration Agency (Japan) 
$80121 \mathrm{~N}$ Uncooled VOx infrared sensor development and application [8012-54]

C. Li, G. D. Skidmore, C. J. Han, DRS Sensors \& Targeting Systems, Inc. (United States)

801210 A self-protecting uncooled microbolometer structure for uncooled microbolometer [8012-126]

Y. Jo, I.-W. Kwon, D. S. Kim, H. B. Shim, H. C. Lee, Korea Advanced Institute of Science and Technology (Korea, Republic of)

$80121 \mathrm{P} \quad$ Electric characteristic of nickel oxide film for the mirobolometer [8012-127]

Y. S. Lee, Kyungpook National Univ. (Korea, Republic of); D. S. Kim, Korea Advanced Institute of Science and Technology (Korea, Republic of); Y.-C. Jung, Kyeongju Univ. (Korea, Republic of); H. C. Lee, Korea Advanced Institute of Science and Technology (Korea, Republic of)

$80121 Q \quad$ VPD PbSe technology fills the existing gap in uncooled low-cost and fast IR imagers [8012-146]

G. Vergara, R. Linares-Herrero, R. Gutiérrez-Álvarez, M. T. Montojo, C. Fernández-Montojo,

A. Baldasano-Ramírez, G. Fernández-Berzosa, New Infrared Technologies, Ltd. (Spain)

8012 IR A detailed analysis for the absorption coefficient of multilevel uncooled infrared detectors [8012-153]

S. E. Kucuk, M. Yusuf Tanrikulu, T. Akin, Middle East Technical Univ. (Turkey)

8012 is A two-stage digital-to-analog converter for bias correction in uncooled microbolometer arrays [8012-154]

A. Toprak, M. Tepegoz, T. Akin, Middle East Technical Univ. (Turkey)

8012 1T A thermal conductance optimization approach for uncooled microbolometers [8012-155]

S. U. Senveli, M. Yusuf Tanrikulu, T. Akin, Middle East Technical Univ. (Turkey)

$80121 \mathrm{U}$ Technical and market trends for microbolometers for thermography and night vision [8012-123]

E. Mounier, Yole Développement (France)

NOTE: See also paper $80123 Z$ in the ORAL STANDBY/POSTERS session.

\section{NIR/SWIR FPAS AND APPLICATIONS}

$80121 \mathrm{~V}$ Dual-band technology on indium gallium arsenide focal plane arrays [8012-55]

P. Dixon, Sensors Unlimited, Inc., part of Goodrich Corp. (United States); C. D. Hess, C. Li, DRS Technologies (United States); M. Ettenberg, J. Trezza, Sensors Unlimited, Inc., part of Goodrich Corp. (United States)

$80121 \mathrm{~W}$ Toward a single-chip TECless/NUCless InGaAs SWIR camera with 120-dB intrinsic operation dynamic range [8012-56]

Y. Ni, B. Arion, Y. M. Zhu, P. Potet, New Imaging Technologies SAS (France); O. Huet,

J. L. Reverchon, J. P. Truffer, J. A. Robo, E. Costard, Alcatel-Thales III-V Lab., Thales Research

\& Technology (France) 
$80121 X \quad$ Recent advances in SWIR MEMS-based tunable Fabry-Pérot microspectrometers [8012-57] J. Antoszewski, T. Nguyen, M. Martyniuk, J. M. Dell, L. Faraone, The Univ. of Western Australia (Australia)

$80121 Y \quad$ Analytic modeling and explanation of ultra-low noise in dense SWIR detector arrays [8012-58]

J. A. Trezza, N. Masaun, M. Ettenberg, Sensors Unlimited, Inc., part of Goodrich Corp. (United States)

$80121 Z$ Modeling of the electrical characteristics of SWIR/MWIR InGaAs/GaAsSb type-II MQW photodiodes [8012-59]

B. Chen, J. Yuan, A. L. Holmes, Jr., Univ. of Virginia (United States)

801220 MOVPE grown InGaAs/GaAsSb type II quantum well photodiode for SWIR focal plane array [8012-60]

H. Inada, H. Mori, Y. Nagai, Y. Iguchi, T. Saitoh, K. Fujii, T. Ishizuka, K. Akita, Sumitomo Electric Industries, Ltd. (Japan)

$801221 \quad$ InGaAs focal plane arrays for low-light-level SWIR imaging [8012-61]

M. MacDougal, A. Hood, J. Geske, J. Wang, F. Patel, D. Follman, J. Manzo, Aerius Photonics, LLC (United States); J. Getty, Raytheon Vision Systems (United States)

801222 IR CMOS: ultrafast laser-enhanced silicon detection [8012-62]

M. U. Pralle, J. E. Carey, H. Homayoon, J. Sickler, X. Li, J. Jiang, D. Miller, C. Palsule, J. McKee, SiOnyx Inc. (United States)

NOTE: See also paper 801240 in the ORAL STANDBY/POSTERS session.

\section{Part 2}

\section{IR OPTICS I}

801223 Somewhere under the rainbow: the visible to far infrared imaging lens [8012-63]

T. A. Palmer, C. C. Alexay, S. Vogel, StingRay Optics, LLC (United States)

801224 Refractive lens design for simultaneous SWIR and LWIR imaging [8012-64]

S. Sparrold, E. Herman, A. Czajkowski, K. O'Shea, Edmund Optics, Inc. (United States)

801225 Compact dual field of view SWIR/MWIR optical system [8012-65]

J. N. Vizgaitis, K. Witte, R. Littleton, P. Perconti, U.S. Army Night Vision \& Electronic Sensors

Directorate (United States)

801226 Optical designs of compact multichannel and all-reflective system for infrared imaging [8012-66]

M. Wang, F. Châteauneuf, C. Proulx, Institut National d'Optique (Canada)

801227 Advanced manufacturing technologies for reduced cost and weight in portable ruggedized VIS-IR multi-mode optical systems for land, sea, and air [8012-67] M. Sweeney, R. Spinazzola, D. Morrison, D. Macklin, J. Marion, General Dynamics Advanced Information Systems (United States) 
801228 Towards infrared DDCA with an imaging function [8012-68]

G. Druart, F. de la Barriere, N. Guérineau, J. Deschamps, ONERA (France); M. Fendler, N. Lhermet, J. Rulliere, CEA-LETI-MINATEC (France); S. Magli, Y. Reibel, SOFRADIR (France); J.-B. Moullec, Direction Générale de L'armement (France)

801229 Compact designs of hyper- or multispectral imagers compatible with the detector dewar [8012-69]

N. Guérineau, G. Druart, F. Gillard, Y. Ferrec, M. Chambon, S. Rommelvère, G. Vincent, R. Haïdar, ONERA (France); J. Taboury, Lab. Charles Fabry, CNRS, Univ. Paris-Sud (France); M. Fendler, CEA-LETI-MINATEC (France)

8012 2A Increasing dynamic range of cameras with dynamic sunlight filter (DSF) [8012-1 10] A. Donval, T. Fisher, D. Cheskis, Y. Ofir, M. Oron, KiloLambda Technologies, Ltd. (Israel)

8012 2B Challenges, constraints, and results of lens design for 17 micron-bolometer focal plane arrays in 8-12 micron waveband [8012-71]

N. Schuster, Umicore Electro-Optic Materials (Belgium); J. Franks, Umicore Coating Services (United Kingdom)

\section{IR OPTICS II}

$80122 \mathrm{C}$ Influence of spinel head window thickness on the performance characteristics of a submarine panoramic infrared imaging system [8012-72]

J. M. Nichols, J. R. Waterman, S. Bayya, J. S. Sanghera, I. D. Aggarwal, U.S. Naval Research Lab. (United States)

$80122 \mathrm{E}$ Advanced manufacturing methods for chalcogenide molded optics [8012-147]

G. Cogburn, LightPath Technologies, Inc. (United States)

8012 2F Development of MWID continuous zoom with large zoom range [8012-134]

M. C. Sanson, J. Cornell, B. Roy, S. Herbert, K. Woodard, K. Sawyer, Corning Inc. (United States)

\section{CRYOCOOLERS FOR IR FOCAL PLANE ARRAYS}

80122 G Development of miniature moving magnet cryocooler SX040 [8012-74]

I. Rühlich, M. Mai, C. Rosenhagen, A. Schreiter, C. Möhl, AIM INFRAROT-MODULE GmbH (Germany)

8012 2H Micro cryogenic coolers for IR imaging (Invited Paper) [8012-75]

R. Lewis, Y. Wang, J. Cooper, M. M. Lin, V. M. Bright, Y. C. Lee, Univ. of Colorado at Boulder (United States); P. E. Bradley, R. Radebaugh, M. L. Huber, National Institute of Standards and Technology (United States)

801221 Adaptation of the low-cost and low-power tactical split Stirling cryogenic cooler for aerospace applications [8012-76]

A. Veprik, S. Zechtzer, N. Pundak, RICOR-Cryogenic \& Vacuum Systems (Israel);

C. Kirkconnell, J. Freeman, Iris Technology Corp. (United States); S. Riabzev, EADS Astrium Ltd. (United Kingdom) 
8012 2J Low vibration microminiature split Stirling cryogenic cooler for infrared aerospace applications [8012-77]

A. Veprik, S. Zechtzer, N. Pundak, RICOR-Cryogenic \& Vacuum Systems (Israel);

C. Kirkconnel, J. Freeman, Iris Technology Corp. (United States); S. Riabzev, EADS Astrium Ltd. (United Kingdom)

8012 2K FLIR systems submicro rotary stirling cycle IDCA for imaging systems [8012-78]

B.-N. Uri, FLIR Systems, Inc. (United States)

8012 2L Release for production of the most compact microcooler in the Thales Cryogenics rotary monobloc range [8012-79]

J.-Y. Martin, S. Freche, R. Griot, Thales Cryogénie S.A. (France); T. Benschop, Thales

Cryogenics BV (Netherlands)

$80122 \mathrm{~N}$ Lifetime testing results and diagnostic performance prediction of linear coolers at Thales Cryogenics [8012-81]

H. van der Weijden, T. Benschop, W. van de Groep, D. Willems, Thales Cryogenics B.V. (Netherlands)

801220 RICOR's new development of a highly reliable integral rotary cooler: engineering and reliability aspects [8012-82]

A. Filis, N. Pundak, M. Barak, Z. Porat, M. Jaeger, RICOR-Cryogenic \& Vacuum Systems (Israel)

$80122 \mathrm{P} \quad$ Stirling-cycle cooler reliability growth at L-3 CE [8012-140]

D. Arndt, D. Kuo, Q. Phan, L-3 Cincinnati Electronics (United States)

HOT - HIGH OPERATING TEMPERATURE FPAS

$80122 \mathrm{Q}$ High-operating temperature MWIR photon detectors based on Type II InAs/GaSb superlattice (Invited Paper) [8012-83]

M. Razeghi, S. A. Pour, E. Huang, G. Chen, A. Haddadi, B.-M. Nguyen, Northwestern Univ. (United States)

8012 2R MWIR InAsSb XBn detector (bariode) arrays operating at 150K (Invited Paper) [8012-84] P. Klipstein, O. Klin, S. Grossman, N. Snapi, I. Lukomsky, M. Brumer, M. Yassen, D. Aronov, E. Berkowitz, A. Glozman, T. Fishman, O. Magen, I. Shtrichman, E. Weiss, SCD Semiconductor Devices (Israel)

801225 InAsSb detectors for visible to MWIR high-operating temperature applications (Invited Paper) [8012-85]

A. I. D'Souza, A. C. Ionescu, M. Salcido, E. Robinson, L. C. Dawson, D. L. Okerlund, DRS Sensors \& Targeting Systems, Inc. (United States); T. J. de Lyon, R. D. Rajavel, H. Sharifi, D. Yap, M. L. Beliciu, S. Mehta, HRL Labs., LLC (United States); W. Dai, G. Chen, Massachusetts Institute of Technology (United States); N. Dhar, Defense Advanced Research Projects Agency (United States); P. Wijewarnasuriya, U.S. Army Research Lab. (United States)

$80122 \mathrm{~T}$ Use of unipolar barriers to block dark currents in infrared detectors (Invited Paper) [8012-86] G. R. Savich, J. R. Pedrazzani, D. E. Sidor, S. Maimon, G. W. Wicks, Univ. of Rochester (United States) 
$80122 \mathrm{U}$ Development of interband cascade infrared photodetectors [8012-87]

Z. Tian, R. Q. Yang, The Univ. of Oklahoma (United States); D. Lubyshev, Y. Qiu,

J. M. Fastenau, W. K. Liu, IQE Inc (United States); J. F. Klem, Sandia National Labs. (United

States); M. B. Johnson, The Univ. of Oklahoma (United States)

8012 2V High operating temperature IR-modules with reduced pitch for SWaP sensitive applications [8012-88]

R. Breiter, J. Wendler, H. Lutz, S. Rutzinger, T. Ihle, J. Ziegler, I. Rühlich, AIM INFRAROT-MODULE GmbH (Germany)

8012 2W HOT infrared detectors using MCT technology [8012-89]

M. Vuillermet, L. Rubaldo, F. Chabuel, C. Pautet, J. C. Terme, SOFRADIR (France); L. Mollard, J. Rothman, N. Baier, CEA-LETI-MINATEC (France)

$80122 \mathrm{X}$ High-operating temperature (HOT) broadband quantum-dot infrared photodetector [8012-90]

P. Vasinajindakaw, X. Lu, X. Qian, S. R. Vangala, W. Goodhue, Univ. of Massachusetts Lowell (United States)

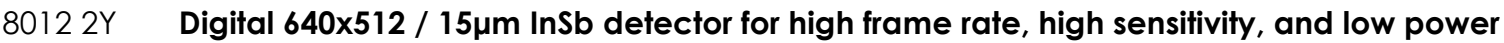
applications [8012-91]

T. Markovitz, I. Pivnik, Z. Calahorra, E. Ilan, I. Hirsh, E. Zeierman, M. Eylon, E. Kahanov, I. Kogan, N. Fishler, M. Brumer, I. Lukomsky, SCD Semiconductor Devices (Israel)

NOTE: See also paper 801241 in the ORAL STANDBY/POSTERS session.

\section{ACTIVE IMAGING I}

801230 New developments in HgCdTe APDs and LADAR receivers [8012-93]

W. McKeag, T. Veeder, J. Wang, M. Jack, Raytheon Co. (United States); T. Roberts, T. Robinson, J. Neisz, C. Andressen, R. Rinker, Raytheon Missile Systems (United States);

T. D. Cook, Naval Air Warfare Ctr. (United States); F. Amzajerdian, NASA Langley Research Ctr. (United States)

\section{ACTIVE IMAGING II}

801232 A 320x256 HgCdTe avalanche photodiode focal plane array for passive and active 2D and 3D imaging [8012-95]

E. de Borniol, P. Castelein, F. Guellec, J. Rothman, G. Vojetta, G. Destéfanis,

CEA-LETI-MINATEC (France); M. Vuillermet, SOFRADIR (France)

HGCDTE

801234 Large format high-operability SWIR and MWIR focal plane array performance and capabilities [8012-97]

J. Bangs, M. Langell, M. Reddy, L. Melkonian, S. Johnson, L. Elizondo, K. Rybnicek, E. Norton, F. Jaworski, J. Asbrock, S. Baur, Raytheon Vision Systems (United States) 
$801235 \quad$ MCT IR detectors in France [8012-98]

G. Destéfanis, CEA-LETI-MINATEC (France); P. Tribolet, M. Vuillermet, D. Billon Lanfrey, SOFRADIR (France)

801236 Latest detector developments with HgCdTe grown by MOVPE on GaAs substrates [8012-99] P. Abbott, P. M. Thorne, C. P. Arthurs, SELEX Galileo Infrared Ltd. (United Kingdom)

801237 The development of 3rd generation IR detectors at AIM [8012-100]

J. Ziegler, D. Eich, M. Mahlein, T. Schallenberg, R. Scheibner, J. Wendler, J. Wenisch,

R. Wollrab, AIM INFRAROT-MODULE GmbH (Germany); V. Daumer, R. Rehm, F. Rutz, M. Walther, Fraunhofer-Institut für Angewandte Festkörperphysik (Germany)

801238 Infrared dual-band detectors for next generation [8012-101]

Y. Reibel, F. Chabuel, C. Vaz, D. Billon-Lanfrey, SOFRADIR (France); J. Baylet, O. Gravrand,

P. Ballet, G. Destefanis, CEA-LETI-MINATEC (France)

801239 Electro-optical characteristics of a MWIR and LWIR planar hetero-structure P+n HgCdTe photodiodes limited by intrinsic carrier recombination processes [8012-102]

R. DeWames, P. Maloney, C. Billman, J. Pellegrino, U.S. Army Night Vision \& Electronic Sensors Directorate (United States)

$80123 \mathrm{~A}$ On the role of dislocations in influencing the electrical properties of HgCdTe photodiodes [8012-148]

R. K. Sharma, Solid State Physics Lab. (India); V. Gopal, Institute of Defence Scientists and Technologists (India); R. S. Saxena, R. K. Bhan, R. Pal, V. Dhar, R. Muralidharan, Solid State Physics Lab. (India)

8012 3B Different approximation for carrier statistic in non-parabolic MWIR HgCdTe photovoltaic devices [8012-144] J. Wang, National Synchrotron Radiation Lab. (China) and Shanghai Institute of Technical Physics (China); X. Chen, W. Hu, L. Wang, Y. Chen, W. Lu, Shanghai Institute of Technical Physics (China); F. Xu, National Synchrotron Radiation Lab. (China)

8012 3C Study of photosensitive area extension in HgCdTe photodiodes using scanning laser microscopy [8012-145]

Y. Chen, W. Hu, X. Chen, J. Wang, W. Lu, Shanghai Institute of Technical Physics (China)

\section{IR OPTICAL MATERIALS}

$80123 D$ Dual-band antireflection coatings on 3rd Gen lenses [8012-103]

T. D. Rahmlow, Jr., J. E. Lazo-Wasem, Rugate Technologies, Inc. (United States); J. N. Vizgaitis, J. Flanagan-Hyde, U.S. Army Night Vision \& Electronic Sensors Directorate (United States)

$80123 \mathrm{~F} \quad$ Visible quality aluminum and nickel superpolish polishing technology enabling new missions [8012-105]

K. G. Carrigan, L-3 Communications Tinsley Labs. Inc. (United States)

NOTE: See also paper 801242 in the ORAL STANDBY/POSTERS session. 
$80123 \mathrm{G}$ A field-deployable spectral reflectometer to characterize surfaces in the infrared from the NIR to the LWIR [8012-107]

L. Moreau, H. Bourque, R. Ouellet, C. Roy, C. Vallières, G. Thériault, ABB Bomem Inc. (Canada)

$80123 \mathrm{H} \quad$ Long wave infrared (8 to 14 microns) hyperspectral imager based on an uncooled thermal camera and the traditional Cl block interferometer (SI-LWIR-UC) [8012-108]

D. Cabib, M. Lavi, A. Gil, U. Milman, Cl Systems (Israel) Ltd. (Israel)

801231 Compact dewar and electronics for large-format infrared detectors [8012-109]

A. Manissadjian, S. Magli, E. Mallet, P. Cassaigne, SOFRADIR (France)

8012 3J Integrated approach to optomechanical system development [8012-111]

T. E. Reney, R. L. Wiggins, L. E. Comstock, J. J. Santman, K. S. Woodard, Corning Inc. (United States)

$80123 \mathrm{~K}$ Classification of thermal face images using radial basis function neural network [8012-112] M. K. Bhowmik, Tripura Univ. (India); D. Bhattacharjee, D. K. Basu, M. Nasipuri, Jadavpur Univ. (India)

$80123 \mathrm{~L}$ The research on infrared small-target detection technology under complex background [8012-113]

L. Liu, X. Wang, J. Chen, Z. Huang, Nanjing Univ. of Science and Technology (China)

$80123 \mathrm{M}$ Characterization of a quantum cascade laser-based emissivity monitor for CORSAIR [8012-141]

M. Lwin, Princeton Univ. (United States) and Space Dynamics Lab. (United States); M. Wojcik, H. Latvakoski, D. Scott, M. Watson, A. Marchant, S. Topham, Space Dynamics Lab. (United States); M. Mlynczak, NASA Langley Research Ctr. (United States)

8012 3N Quantum cascade laser as a mid-infrared photovoltaic and photoconductive detector [8012-142]

X. Chen, D. Shyu, F.-S. Choa, Univ. of Maryland, Baltimore County (United States); S. Trivedi, Brimrose Corp. of America (United States)

\section{VARIOUS UNCOOLED DETECTOR TECHNOLOGIES I}

$801230 \quad$ Further applications for mosaic pixel FPA technology [8012-114]

K. C. Liddiard, Electro-optic Sensor Design (Australia)

$80123 \mathrm{P}$ Towards very high-resolution infrared camera core [8012-115]

L. Le Noc, D. Dufour, M. Terroux, B. Tremblay, J. Lambert, L. Mercier, M. Morissette, C. Vachon, INO (Canada); D. Tang, Canadian Special Operations Command (Canada); A. Bergeron, INO (Canada)

$80123 Q \quad$ A look at non-uniformity correction in the spatial frequency domain [8012-17]

G. Raz, Y. Weiss, Elbit Systems Electro-Optics El-Op Ltd. (Israel) 
$80123 R \quad$ Development of noncryogenic cooled carbon nanotube-based infrared focal plane array with integrated readout circuitry [8012-116]

N. Xi, K. W. C. Lai, H. Chen, L. Chen, C. K. M. Fung, Michigan State Univ. (United States)

\section{VARIOUS UNCOOLED DETECTOR TECHNOLOGIES II}

$801235 \quad$ New materials for uncooled IR imaging: nickel manganite thin films grown by spin spray [8012-117]

S. W. Ko, J. Li, M.-Y. Lee, The Pennsylvania State Univ. (United States); E. Dickey, North Carolina State Univ. (United States); T. Jackson, S. Trollier-McKinstry, The Pennsylvania State Univ. (United States)

8012 3T Microstructure of vanadium oxide used in microbolometers [8012-118]

B. D. Gauntt, J. Li, O. M. Cabarcos, H. A. Basantani, C. Venkatasubramanian,

S. S. N. Bharadwaja, N. J. Podraza, T. N. Jackson, D. L. Allara, The Pennsylvania State Univ. (United States); S. Antrazi, 4Wave Inc. (United States); M. W. Horn, The Pennsylvania State Univ. (United States); E. C. Dickey, North Carolina State Univ. (United States)

$80123 \mathrm{U}$ Thin film silicon and germanium for uncooled microbolometer applications [8012-119]

D. B. Saint John, H.-B. Shin, M.-Y. Lee, The Pennsylvania State Univ. (United States);

E. C. Dickey, North Carolina State Univ. (United States); N. J. Podraza, The Pennsylvania State Univ. (United States) and Univ. of Toledo (United States); T. N. Jackson, The Pennsylvania State Univ. (United States)

$80123 V$ A 256-pixel pyroelectric linear array with new black coating [8012-120] V. Norkus, Technische Univ. Dresden (Germany) and DIAS Infrared GmbH (Germany); M. Schossig, G. Gerlach, Technische Univ. Dresden (Germany); R. Köhler, DIAS Infrared GmbH (Germany)

$80123 \mathrm{~W} \quad$ Small pitch high performance thermopile focal plane arrays [8012-121] D. Kryskowski, UD Holdings LLC (United States)

$80123 Y$ Amorphous InSb and InAs0.3Sbo.7 for long wavelength infrared detection [8012-128] T. Zens, P. Becla, A. M. Agarwal, L. C. Kimerling, Massachusetts Institute of Technology (United States); A. Drehman, Air Force Research Lab. (United States)

\section{ORAL STANDBY/POSTERS}

$80123 Z$ Impacts and mitigation strategies of sun exposure on uncooled microbolometer image sensors [8012-149]

D. Dorn, O. Herrera, C. Tesdahl, E. Shumard, Y.-W. Wang, Schneider Electric (United States)

801240 Characterization of SiGe-detector arrays for visible-NIR imaging sensor applications [8012-151]

A. K. Sood, R. A. Richwine, A. W. Sood, Y. R. Puri, Magnolia Optical Technologies, Inc. (United States); N. DiLello, J. L. Hoyt, T. I. Akinwande, Massachusetts Institute of Technology (United States); N. Dhar, Defense Advanced Research Projects Agency (United States); R. S. Balcerak, Ray S. Balcerak, LLC (United States); T. G. Bramhall, U.S. Army Aviation and Missile Command (United States) 
801241 Characterization of different transitions in quantum dots-in-a-well (DWELL) infrared photodetectors [8012-156]

A. V. Barve, J. O. Kim, Y. D. Sharma, T. Rotter, S. Sengupta, J. Montoya, S. Krishna, The Univ. of New Mexico (United States)

801242 Erosion resistant anti-reflection coating for ZnSe, CZnS, chalcogenide, and glass substrates [8012-158]

K. M. Khajurivala, Janos Technology (United States)

801243 Surface study of thioacetamide and zinc sulfide passivated long wavelength infrared type-II strained layer superlattice [8012-159]

K. Banerjee, J. Huang, S. Ghosh, R. XU, C. G. Takoudis, Univ. of Illinois at Chicago (United States); E. Plis, S. Krishna, The Univ. of New Mexico (United States); S. Ketharanathan, M. Chriss, EPIR Technologies, Inc. (United States)

Author Index 
Downloaded From: https://www.spiedigitallibrary.org/conference-proceedings-of-spie on 26 Apr 2023

Terms of Use: https://www.spiedigitallibrary.org/terms-of-use 


\title{
Conference Committee
}

\author{
Symposium Chair
}

William Jeffrey, HRL Laboratories, LLC (United States)

Symposium Cochair

Kevin P. Meiners, Office of the Secretary of Defense (United States)

Conference Chairs

Bjørn F. Andresen, SCD Semiconductor Devices (Israel)

Gabor F. Fulop, Maxtech International, Inc. (United States)

Paul R. Norton, U.S. Army Night Vision \& Electronic Sensors Directorate (United States)

Program Committee

Christopher C. Alexay, StingRay Optics, LLC (United States)

Timothy Ashley, QinetiQ Ltd. (United Kingdom)

J. Bajaj, Teledyne Imaging Sensors (United States)

Stefan T. Baur, Raytheon Vision Systems (United States)

Philippe F. Bois, Alcatel-Thales III-V Laboratory (France)

Wolfgang A. Cabanski, AIM INFRAROT-MODULE GmbH (Germany)

John T. Caulfield, Cyan Systems (United States)

John W. Devitt, Georgia Tech Research Institute (United States)

Nibir K. Dhar, Defense Advanced Research Projects Agency (United

States)

Michael T. Eismann, Air Force Research Laboratory (United States)

Martin H. Ettenberg, Sensors Unlimited, Inc., part of Goodrich Corp.

(United States)

Sarath D. Gunapala, Jet Propulsion Laboratory (United States)

Charles M. Hanson, L-3 Electro-Optical Systems (United States)

Masafumi Kimata, Ritsumeikan University (Japan)

Hee Chul Lee, Korea Advanced Institute of Science and Technology

(Korea, Republic of)

Paul D. LeVan, Air Force Research Laboratory (United States)

Chuan C. Li, DRS Technologies, Inc. (United States)

Wei Lu, Shanghai Institute of Technical Physics (China)

Paul L. McCarley, Air Force Research Laboratory (United States)

R. Kennedy McEwen, SELEX Galileo Ltd. (United Kingdom)

John L. Miller, FLIR Systems, Inc. (United States)

A. Fenner Milton, U.S. Army RDECOM CERDEC Night Vision \& Electronic Sensors Directorate (United States) 
Peter W. Norton, BAE Systems (United States)

Joseph G. Pellegrino, U.S. Army Night Vision \& Electronic Sensors

Directorate (United States)

Ray Radebaugh, National Institute of Standards and Technology (United States)

Manijeh Razeghi, Northwestern University (United States)

Colin E. Reese, U.S. Army Night Vision \& Electronic Sensors Directorate (United States)

Ingmar G. Renhorn, Swedish Defence Research Agency (Sweden)

Antoni Rogalski, Military University of Technology (Poland)

Ingo Rühlich, AIM INFRAROT-MODULE GmbH (Germany)

Gabby Sarusi, Elbit Systems Electro-Optics ElOp Ltd. (Israel)

Piet B. W. Schwering, TNO Defence, Security and Safety (Netherlands)

Itay Shtrichman, SCD Semiconductor Devices (Israel)

Rengarajan Sudharsanan, Boeing Spectrolab, Inc. (United States)

Stefan P. Svensson, U.S. Army Research Laboratory (United States)

Venkataraman S. Swaminathan, U.S. Army Armament Research,

Development and Engineering Center (United States)

Simon Thibault, Université Laval (Canada)

Meimei Z. Tidrow, U.S. Army Night Vision \& Electronic Sensors

Directorate (United States)

Jean-Luc M. Tissot, ULIS (France)

Philippe Tribolet, SOFRADIR (France)

Jay N. Vizgaitis, U.S. Army Night Vision \& Electronic Sensors Directorate (United States)

James R. Waterman, U.S. Naval Research Laboratory (United States)

Lucy Zheng, Institute for Defense Analyses (United States)

Session Chairs

$1 \quad$ Target Acquisition with Today's Leading Imaging Technologies

Rainer Breiter, AIM INFRAROT-MODULE GmbH (Germany)

2 Threat Identification I

Mario O. Münzberg, Carl Zeiss Optronics GmbH (Germany)

3 Threat Identification II

Ingmar G. Renhorn, Swedish Defence Research Agency (Sweden)

$4 \quad$ Smart Image and Signal Processing

Paul L. McCarley, Air Force Research Laboratory (United States)

John T. Caulfield, Cyan Systems (United States)

5 QWIP and QDIP

Sarath D. Gunapala, Jet Propulsion Laboratory (United States) 
Type II Superlattice FPAs I

Meimei Z. Tidrow, U.S. Army Night Vision \& Electronic Sensors

Directorate (United States)

Manijeh Razeghi, Northwestern University (United States)

Lucy Zheng, Institute for Defense Analyses (United States)

$7 \quad$ Type II Superlattice FPAs II

Meimei Z. Tidrow, U.S. Army Night Vision \& Electronic Sensors Directorate (United States)

Manijeh Razeghi, Northwestern University (United States)

Lucy Zheng, Institute for Defense Analyses (United States)

$8 \quad$ Emerging Uncooled Technologies

Colin E. Reese, U.S. Army Night Vision \& Electronic Sensors

Directorate (United States)

Charles M. Hanson, L-3 Electro-Optical Systems (United States)

9 Uncooled FPAs and Applications I

Jean-Luc M. Tissot, ULIS (France)

Avraham Fraenkel, SCD Semiconductor Devices (Israel)

10 Joint Keynote Session with Conference 8014

Paul R. Norton, U.S. Army Night Vision \& Electronic Sensors

Directorate (United States)

11 Uncooled FPAs and Applications II

Masafumi Kimata, Ritsumeikan University (Japan)

Stefan T. Baur, Raytheon Vision Systems (United States)

12 NIR/SWIR FPAs and Applications

Martin H. Ettenberg, Sensors Unlimited, Inc., part of Goodrich

Corporation (United States)

13 IR Optics I

Jay N. Vizgaitis, U.S. Army Night Vision \& Electronic Sensors Directorate (United States)

Christopher C. Alexay, StingRay Optics, LLC (United States)

14 IR Optics II

Christopher C. Alexay, StingRay Optics, LLC (United States)

Jay N. Vizgaitis, U.S. Army Night Vision \& Electronic Sensors Directorate (United States) 
15 Cryocoolers for IR Focal Plane Arrays

Alexander Veprik, RICOR-Cryogenic \& Vacuum Systems (Israel) Ingo Rühlich, AIM INFRAROT-MODULE GmbH (Germany)

Ray Radebaugh, National Institute of Standards and Technology (United States)

16 HOT - High Operating Temperature FPAs

Michael T. Eismann, Air Force Research Laboratory (United States)

Stuart B. Horn, U.S. Army Night Vision \& Electronic Sensors Directorate (United States)

17 Active Imaging I

R. Kennedy McEwen, SELEX Galileo Ltd. (United Kingdom)

18 Active Imaging II

R. Kennedy McEwen, SELEX Galileo Ltd. (United Kingdom)

$19 \quad \mathrm{HgCdTe}$

Philippe Tribolet, SOFRADIR (France)

Joseph G. Pellegrino, U.S. Army Night Vision \& Electronic Sensors

Directorate (United States)

Michel Vuillermet, SOFRADIR (France)

20 IR Optical Materials

Jay N. Vizgaitis, U.S. Army Night Vision \& Electronic Sensors

Directorate (United States)

21 Application of Selected Technologies

John L. Miller, FLIR Systems, Inc. (United States)

Bjørn F. Andresen, SCD Semiconductor Devices (Israel)

22 Various Uncooled Detector Technologies I

John L. Miller, FLIR Systems, Inc. (United States)

Paul R. Norton, U.S. Army Night Vision \& Electronic Sensors

Directorate (United States)

23 Various Uncooled Detector Technologies II

John L. Miller, FLIR Systems, Inc. (United States)

Bjørn F. Andresen, SCD Semiconductor Devices (Israel) 


\section{Introduction}

The Thirty-Seventh conference on Infrared Technology and Applications was held the week of April 25-29. 2011 at the Orlando World Center Marriott Resort and Convention Center in Orlando, Florida. The agenda was divided into 23 sessions:

1. Target acquisition with today's leading imaging technologies

2. Threat identification I

3. Threat identification II

4. Smart imagining and signal processing

5. QWIP and QDIP

6. Type II Superlattice FPAs I

7. Type II Superlattice FPAs II

8. Emerging uncooled technologies

9. Uncooled FPAs and applications I

10. Keynote-Wide-area infrared surveillance: performance requirements and technology needs

11. Uncooled FPAs and applications II

12. NIR/SWIR FPAs and applications

13. IR Optics I

14. IR Optics II

15. Cryocoolers for IR Focal Plane Arrays

16. HOT - High Operating Temperature FPAs

17. Active imaging I

18. Active imaging II

19. $\mathrm{HgCdTe}$

20. IR optical materials

21. Application of selected technologies

22. Various uncooled detector technologies I

23. Various uncooled detector technologies II

In addition, there were thirty poster papers presented for discussion on Thursday evening - these have been added to the 23 sessions in the Proceedings. High lights of six topical areas are summarized below:

- Target acquisition and threat identification

- Optics

- Smart image and signal processing

- Uncooled thermal detectors

- Photon detectors

- Cryocoolers

\section{Target Acquisition / Threat Identification}

Detection, recognition and identification of objects relevant to defense and security were discussed in sessions $1-3$ and 21. The scenarios varied from faces at a few meters distance to Earth observation from satellites. Focus was put on demonstrating application of advanced technologies, techniques and design meth ods in new systems mounted on various platforms.

Integration of state-of-the art broadband $\mathrm{InSb}$ or $\mathrm{HgCdTe}$ FPAs in a compact long range target acquisition thermal imager was demonstrated. Cooled detectors having $1280 \times 1024$ pixels were used and target identification was predicted for ranges up to 7 $\mathrm{km}$. While discussing the design of the imager, a "visualization tool" was described. The tool is a holistic simulation of the thermal imager which starts from using high resolution visible images.

These long-range imaging systems employ cryogenically cooled FPAs. Several uncooled microbolometer FPAs were presented and simulation was used to compare their performance in systems with that of state of the art $2^{\text {nd }}$ generation, scanning, cooled LWIR arrays. It was shown that similar recognition ranges may be achieved for the two types of systems.

Fast spectral scans are frequently required for detection and identification of targets. This is the case when the target or sensor system moves fast, the signature changes rapidly, as during an explosion, or in the presence of turbulence. Several systems employing fast spectral imaging were outlined. One demonstrated the marriage of $3^{\text {rd }}$ generation FPAs and micro-optical diffractive lens arrays. This system allows target ID via simultaneous spectral investigation.

Defense and security agencies are in constant search for new ways of detecting chemical and biological threats used by terrorist organizations. One research institute demonstrated their four-channel SWIR/ MWIR radiometer for simultaneous four band measurements with a rise time of $6 \mu \mathrm{sec}$. The measured 
data may be used for identifying explosives and providing kill assessment.

While this system investigated explosions, another system was demonstrated which detects highly energetic materials (HEM) and homemade explosives (HME) prior to explosion at stand-off ranges as long as 60 meters. The high spectral resolution was provided by use of a MWIR Fourier Transform interferometer. Both passive and active modes of operation were demonstrated.

High reliability infrared detectors were discussed for space applications. These detectors cover various kinds of applications like Visible to VLWIR hyperspectral observation of the earth's atmosphere for meteorological or scientific purposes.

An airborne thermal infrared imaging spectrometer with 128 bands in the LWIR window was presented. Results from flight trials were shown.

It is known from measurements that the identification range advantage obtained by MWIR, as opposed to LWIR, may be wiped out in the presence of strong turbulence. Turbulence compensation is well known in astronomy. Can the same techniques be used in ground-to-ground scenarios? One laboratory suggested a method based on multiple time-separated images. It was demonstrated in simulations that the method has the potential of providing turbulence-free images and improved long range target identification-Fig. 1 .

Recognition of human faces at public places like airports is a very important security-related means for apprehension of terrorists. Variation of illumination and lack of same hampers face recognition in the vis-
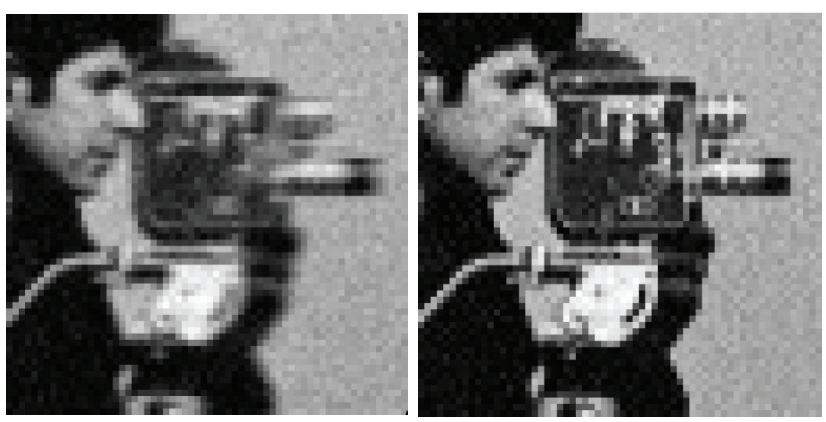

Fig. 1. Defocused input image (left) and processed image (right). Defocusing simulates turbulence. ible spectrum. Veins and tissues give rise to a unique thermal pattern which is different even for identical twins. Two universities have collaborated on the development and implementation of an affordable, robust and accurate security system based on facial thermograms. Presented experimental results showed an average recognition success rate of $94 \%$.

\section{Optics}

Presentations in the three IR optics sessions, 13, 14 and 20, showed a strong effort by the optics community to answer the military's requirement for simultaneous operation in two or more of the spectral bands traditionally called "atmospheric windows". The use of multiple band operation facilitates target detection and identification. These systems are also required to be compact and light.

Which is the optimal type of a multiband optical sys-tem-refractive or reflective? Last year this conference heard a presentation of an essentially reflective multiband system which used folded optics in order to attain good compactness. This design concept was challenged in the present conference by a refractive system which was claimed to operate simultaneously over a large number of bands between visible and LWIR. The optimum combination of optical materials is determined by use of numerical methods to evaluate the dispersive properties of materials in terms of both instantaneous values and volatilities.

Several multiband optics designs were presented. One presentation discussed the design and development of a dual field of view, all-refractive, infrared optical system that images the MWIR radiation in one field of view and the SWIR in a narrower second field of view, both onto the same detector. Another walked the attendees through the selection of materials for a refractive system operating simultaneously in the SWIR and LWIR bands. A third presentation demonstrated a novel, all-reflective, off-axis optical systems for applications in space. Two designs are shown in Figure 2.

A group of laboratories reported on a cooperative effort to incorporate wide fov imaging functions, including compact and robust hyper- and multi-spectral imagers, inside a cooled detector dewar. This minia- 

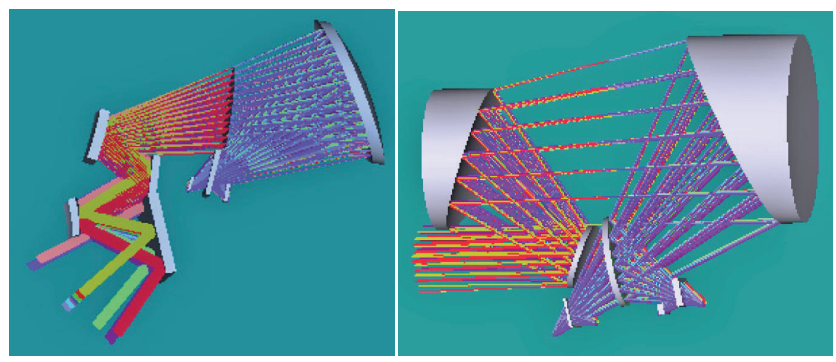

Fig. 2. Multi-band optical designs using three powered optical mirrors. The systems have two (left) and four (right) FPAs.

turization gives imaging capabilities to vehicles with small payload capacities. The stable low temperature improves the system sensitivity. First experimental results were presented from two spectral system architectures based on new optical designs. One of these is illustrated in Fig. 3.

The detector is often considered the "heart" of the system. To protect this vital component and its signal processor from intense blinding radiation from natural sources like the sun or from countermeasure sources. one paper presented a protection filter which operates on the exposed FPA pixels and returns to a neutral state when exposure is terminated.

Development of opto-mechanical systems requires strong communication between the various engineering and manufacturing functions. One company discussed their method and software for tightly integrating optical and mechanical design and fabrication and testing. Several examples were presented showing important gains in rapid and effective development.

One presentation focused on antireflection coatings for multi-lens systems with simultaneous operation in the MW- and LW-bands. A 10-element system with 95\% AR transmittance per lens in each band will have an overall transmittance of about $60 \%$. Design. coating, and characterization of high Dual Band Anti-

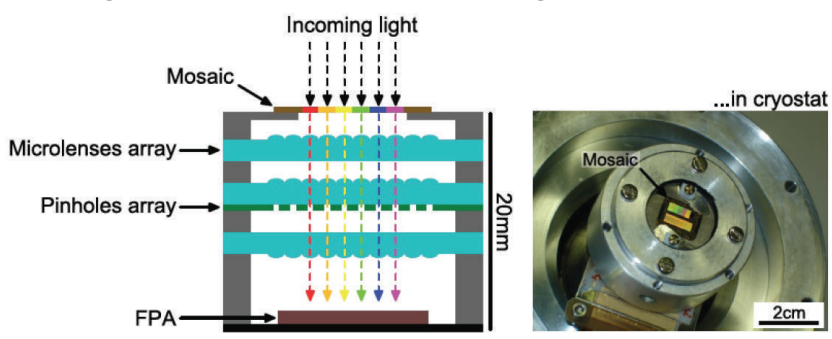

Fig. 3. (a) Compact multispectral camera integrated in cold shield. (b) Seen in opened laboratory dewar.
Reflection, DBAR, films for a $3^{\text {rd }}$ Gen set of lenses were demonstrated. Dual band and broadband trans.mittances were compared.

\section{Smart image and signal processing}

Session 4 on smart image and signal processing included papers featuring readouts with on-chip processing to enhance the functionality of the FPA. A variety of feature options were described in separate papers, including:

- multi-resolution and multi-scale imaging

- combining thermal imaging with laser rangefinder functions

- designs to overcome reset noise in CTIA pixels

- calibration of polarimeter FPAs

- hexagonal pixel layout FPAs and related signal processing functions

Figure 4 illustrates the angular output before and after calibration of a four-pixel group that have polarizer grids (vertical, horizontal, and $\pm 45^{\circ}$ orientation) over the pixels.

The difference of Gaussians was used to extract feature edges in an array of pixels from local contrast differences as illustrated in Fig. 5.
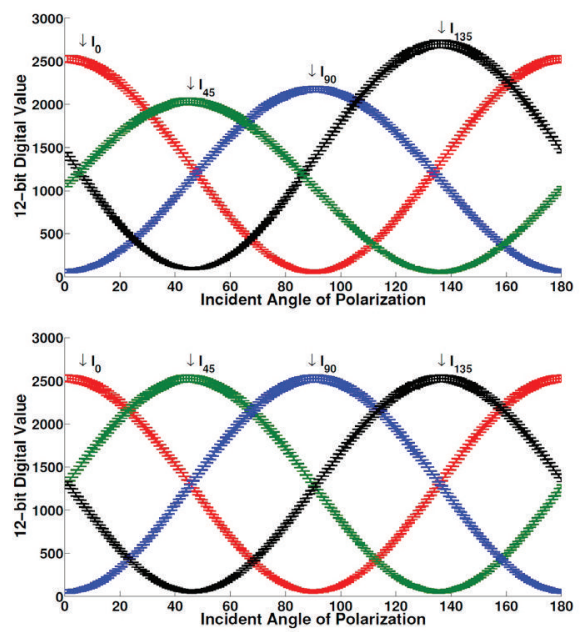

Figure 4. Average response of four polarization filter orientations before (top) and after (bottom) calibration. 


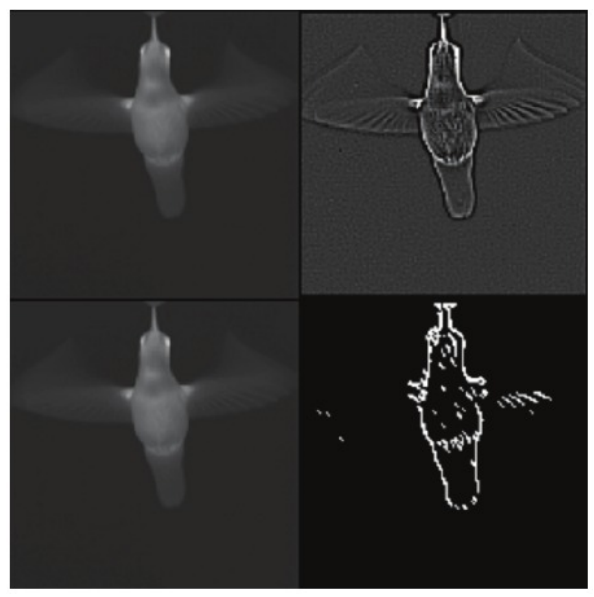

Fig. 5 The difference of Gaussian response of a humming bird shows enhanced edge contrast as shown in the upper-right panel (input scene is in the upper-left). The zero-crossing image as shown in the lower-right image preserves the essential geometries in the scene, but conveys the information in a 1-bit representation. (Infrared imagery courtesy of the SE-IR Corporation, Goleta, CA)

\section{Uncooled thermal detectors}

Sessions 8, 9, and 11, 22, and 23 discussed uncooled detectors. The two leading uncooled technologies -- vanadium oxide $\left(\mathrm{VO}_{\mathrm{x}}\right)$ and amorphous silicon microbolometers are continuing to be rapidly improved. Development of $17 \mu \mathrm{m}$ pixel pitch FPAs is being extended to both smaller arrays $-320 \times 240$ - and arrays larger than 3 Mpixel-2048 x 1536. Currently. the largest such array - made by a U.S. company - is shown on a wafer in Fig. 6.

In addition, development of sub- $17 \mu \mathrm{m}$ pixel FPAs is continuing in the U.S., France, Japan and Sweden.

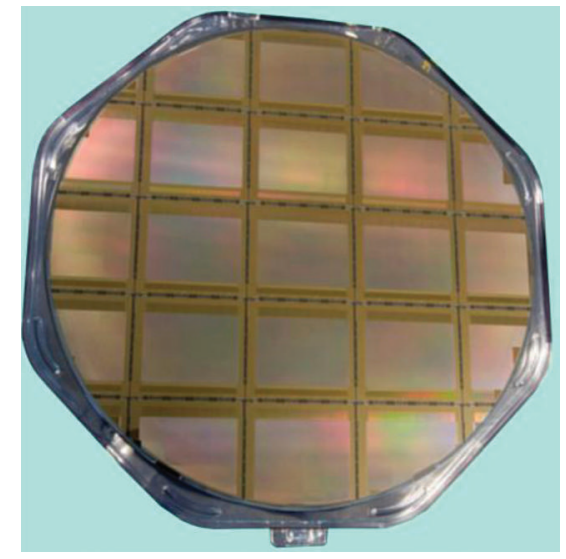

Fig. 6. $2048 \times 1536$ uncooled $\mathrm{VO}_{\mathrm{x}}$ microbolometers with $17 \mu \mathrm{m}$ pixel pitch on a $200 \mathrm{~mm}$ wafer.
Two Japanese companies have made advances in uncooled SOI (silicon-on-insulator) technology in which change in the forward bias voltage of silicon diodes with temperature is used to sense the incident infrared radiation. One company showed that the technology could be pushed to pixel sizes as small as $12 \mu \mathrm{m}$. Another company improved its SOI process by using $0.13 \mu \mathrm{m}$ CMOS design rules and by placing micro-holes in the active cell that reduce the thermal capacity (but which do not decrease the absorption since they are smaller than the infrared wavelengths). This process is now believed ready for volume production. Figure 7 shows an image formed with this $320 \times 240$ SOI FPA with $22 \mu \mathrm{m}$ pixels.

Sub-17 $\mu \mathrm{m}$ pixel FPA design has also been included in the analysis of ultra-low-cost PIR sensors for home security by an Australian company

The development of novel uncooled detectors is also continuing. The following novel uncooled technologies were reported on in this conference:

- A photomechanical imager (an array of bimaterial microcantilevers that are read out optically) which was demonstrated as a Hostile Fire Indicator in the MWIR.

- A microbolometer based on single-crystal $\mathrm{Si} /$ SiGe quantum wells in which $17 \mu \mathrm{m}$ pixel structures were demonstrated.

- A novel read-out for thermopile focal plane arrays that modeling shows can compete with $25 \mu \mathrm{m}, 17 \mu \mathrm{m}$ and $12 \mu \mathrm{m}$ pitch microbolometers in both NE $\Delta \mathrm{T}$ and thermal response time.

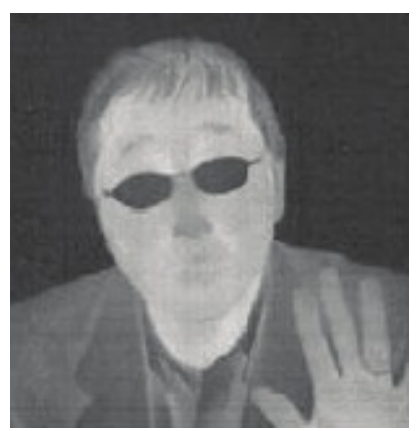

Fig. 7 Low-cost $320 \times 240$ SOI FPA. 


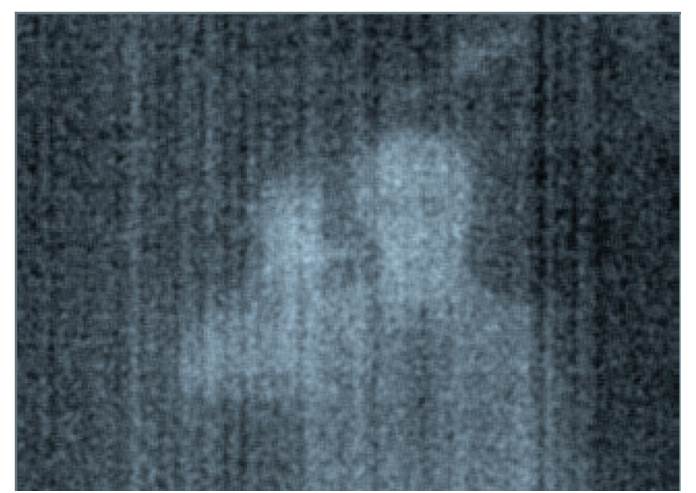

Fig. 8 Passive Terahertz image taken with an altered 320 x $240 \mathrm{VO}_{\mathrm{x}}$ microbolometer-based "Handy THz camera".

In a related area, researchers in Japan demonstrated improved passive and active Terahertz imaging by altering a $320 \times 240 \mathrm{VO}_{\mathrm{x}}$ microbolometer for use as a Terahertz sensor. Figure 8 shows a passive Terahertz image that has been integrated over 64 frames and a subarray of $3 \times 3$ pixels.

\section{Photon detectors}

Photon detector presentations continued to report good progress across the spectrum of device technolo. gies in sessions 5-7, 10, 12, and 16-19.

QWIP and QDIP technologies reported new milestones in development. QWIP activities are inspired by NASA's plans to launch the LANDSAT Data Continuity Mission with QWIP detectors providing the LWIR sensors - see Fig. 9. This has focused development on large format $-512 \times 640$ - broadband QWIPs in the spectral range of $10-$ to $-13 \mu \mathrm{m}$. The spectral

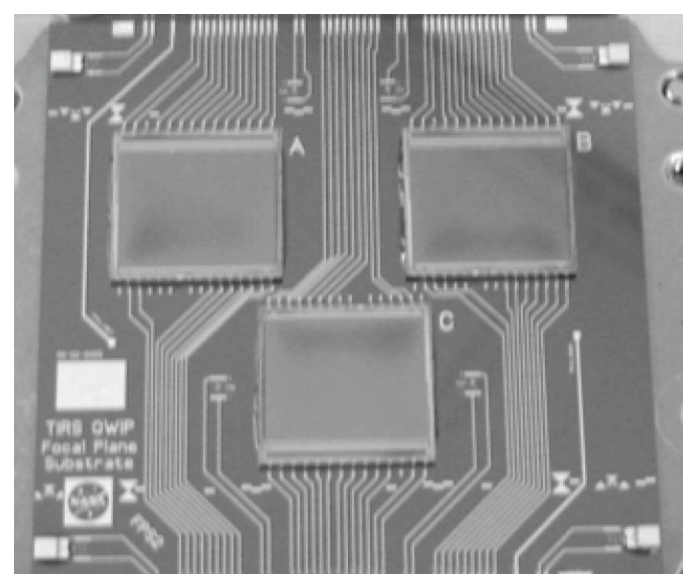

Fig. 9 The Thermal Infrared Sensor (TIRS) focal plane showing three QWIP sensor-chip assemblies.
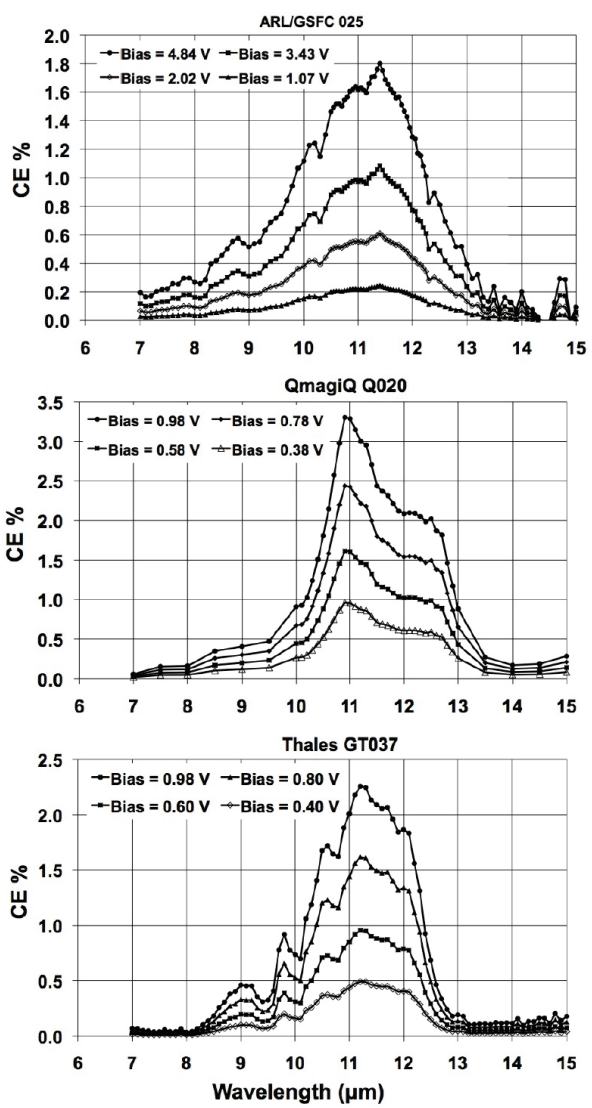

Fig. 10 Collection efficiency (CE) of QWIP FPAs from three NASA vendors as a function of wavelength for several bias values.

collection efficiency - product of quantum efficiency $x$ photoconductive gain - for three of NASA's QWIP vendors are illustrated in Fig. 10 at a temperature a few degrees below the planned operating temperature of $43 \mathrm{~K}$. The QWIP arrays all were measured to have $\mathrm{NE} \Delta \mathrm{T}$ values between 15 and $17 \mathrm{mK}$ with the f/1.64 instrument.

Type II superlattice FPAs provided an update on this rapidly advancing detector technology. Typical Type II superlattice FPAs have about 6x higher leakage (or dark) current compared to $\mathrm{HgCdTe}$ (Rule 7). The separation between the two detector materials performance has been reduced in recent years from a signifi. cantly wider margin however. Improvements in Type II materials are partly responsible for narrowing the performance gap, as illustrated in Fig. 11 that shows how the lattice quality has been improved, as X-ray rocking curve measurements show. 


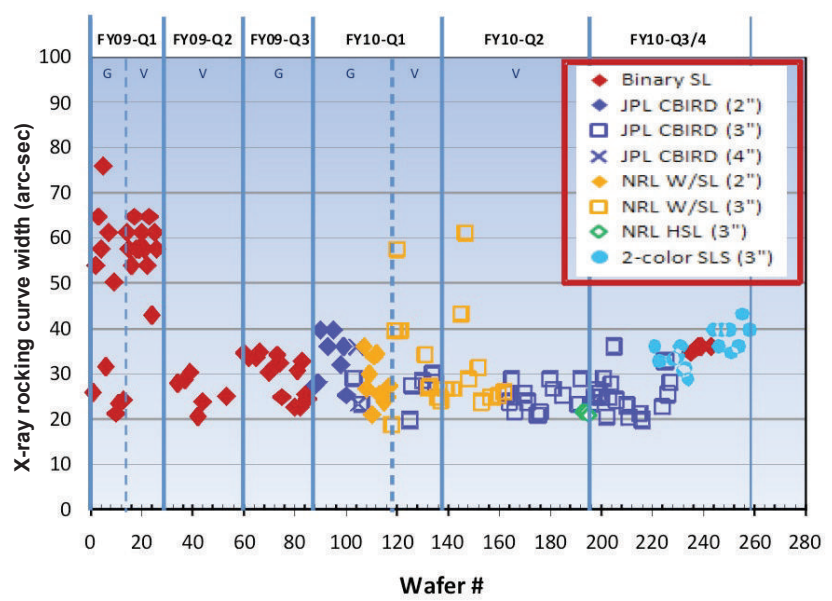

Fig. 11 X-ray crystal rocking curve data shows significant crystal quality improvement as experience is gained in growing Type II SLS material

Arrays of Type II superlattices of $1 \mathrm{~K} \times 1 \mathrm{~K}$ have been demonstrated-see Fig. 12 for one from this conference-and a variety of barrier structures have been used to balance optical absorption and recombination mechanisms and for control of leakage currents.

Other Type II superlattice development efforts were described on precise inductively-coupled plasma (ICP) etching, dual band devices with reduced pixel size-see Fig. 13-growth on alternative substrates (GaAs), and the development of large, 4-inch GaSb substrates. Techniques for backside removal of the $\mathrm{GaSb}$ to eliminate parasitic absorption were reported as well.

A major issue in Type II materials has been the minority-carrier lifetime-typically measured in the range

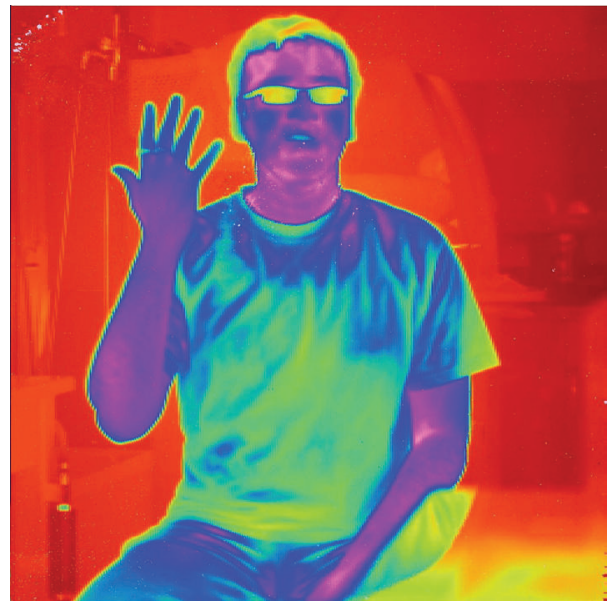

Fig. 12 Image taken with a $1 \mathrm{~K} \times 1 \mathrm{~K}$ Type II superlattice FPA.

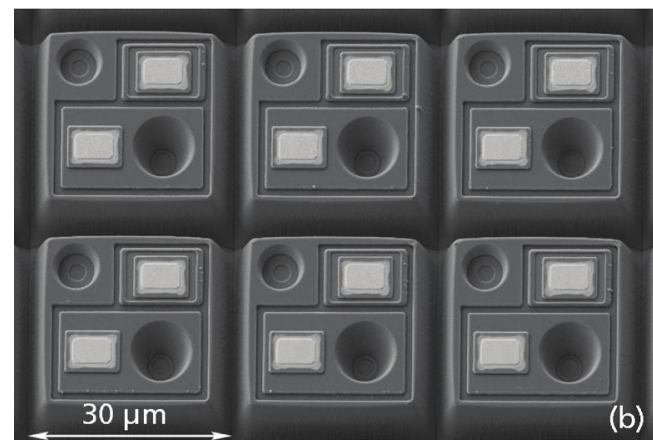

Fig. 13 Dual-band MWIR/MWIR Type II superlattice pixels with reduced $-30 \mu \mathrm{m}-$ pixel size.

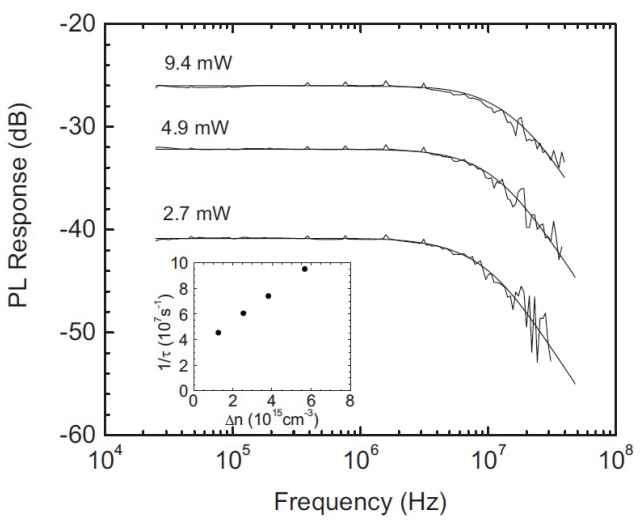

Fig. 14 Photoluminescence-decay measurements made on an LWIR Type II superlattice sample showing a lifetime of $31 \mathrm{nsec}$ for the low-flux limit of photo-excitation.

of about $75 \mathrm{nsec}$ for MWIR materials and $20 \mathrm{nsec}$ for LWIR-effective bandgaps-see Fig. 14-compared with $10 \mu \mathrm{sec} / 2 \mu \mathrm{sec}$ for MWIR/LWIR HgCdTe materials. A possible explanation related to the higher LO phonon energy in InAs and $\mathrm{GaSb}-\sim 30 \mathrm{meV}-\mathrm{com}$ pared to $\mathrm{HgCdTe}$ with $\sim 17 \mathrm{meV}$ was presented.

Short wavelength infrared (SWIR) sensors continue to be under active development-see Fig. 15. A program to combine a SWIR array with $10 \mu \mathrm{m}$ pixels together with an uncooled LWIR array with $20 \mu \mathrm{m}$ pixels was presented. Such a combination would allow sensor fusion of reflected and emitted light to cover a wide variety of situations that soldiers may encounter, including the ability to see laser beams and thermal signatures combined.

Wide SWIR dynamic range $-120 \mathrm{db}$-is available with InGaAs arrays by operating the array in forward bias like a solar cell-the open-circuit voltage being generated by the photon flux. The open-circuit voltage 

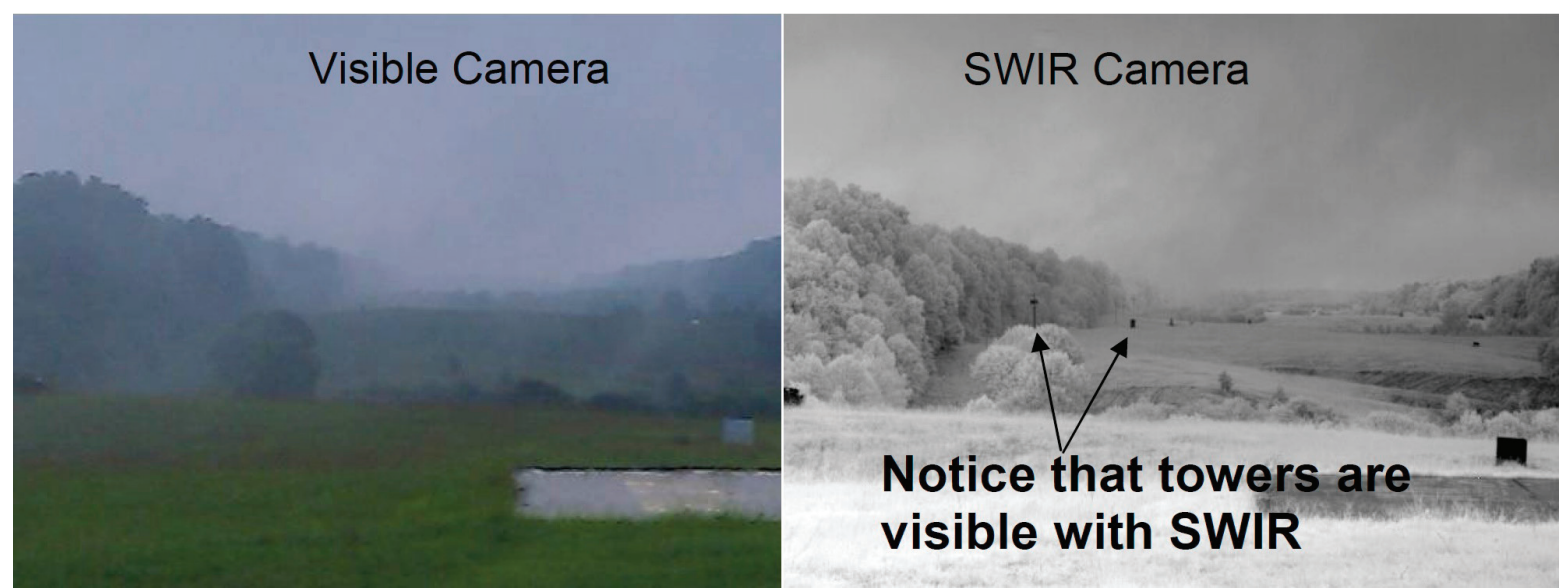

Fig. 15 Comparison of visible and SWIR imagery in atmospheric fog.

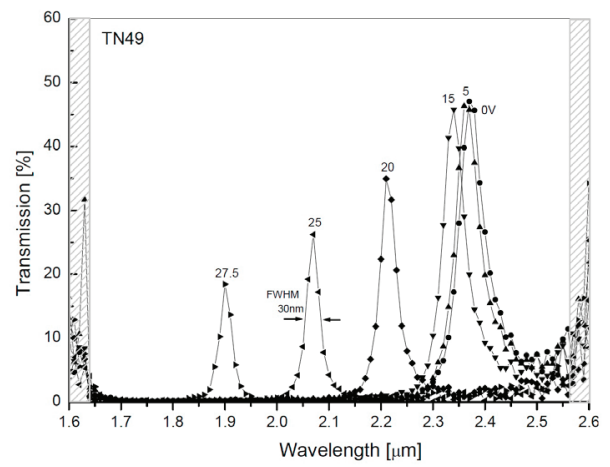

Fig. 16 Tuning range of a MEMs Fabry-Perot structure from 1.9 to $2.4 \mu \mathrm{m}$ with applied voltage noted above peaks.

increases only logarithmically with the photocurrent Such sensors are being proposed as an alternative to uncooled arrays for automotive applications such as extending the visibility range.

Pixel-level MEMs Fabry-Perot arrays were described with tuning in the SWIR band as illustrated in Fig. 16.

Progress in reducing the dark current and noise in dense arrays was reported. The trade-off in power requirements for TE cooling vs. using SWIR illuminators was also discussed. In addition to InGaAs SWIR detectors, other approaches were described based on MOVPE Type II materials and "black" silicon.

The conference keynote session described the requirements and technology needs for wide-area persistent surveillance sensors.

A half-day session was devoted to High-Operating Temperature (HOT) detectors - a hot topic for the

past couple of years. Papers presented in this section covered a variety of detector material candidates, including:

- MWIR Type II superlattices

- MWIR InAsSb xBn structures

- InAsSb alloys with special absorber structures

- Interband cascade photodetectors

- $\mathrm{HgCdTe}$ photodiodes

- Quantum dot (Qdot or QDIP) photodetectors

- $\mathrm{InSb}$

Progress with detectors having majority-carrier barriers is extremely active-see Fig. 17. The paper on Type II superlattices in this session reported designs that included such a barrier, as did other papers more specific to this approach. Barrier detectors have a po-

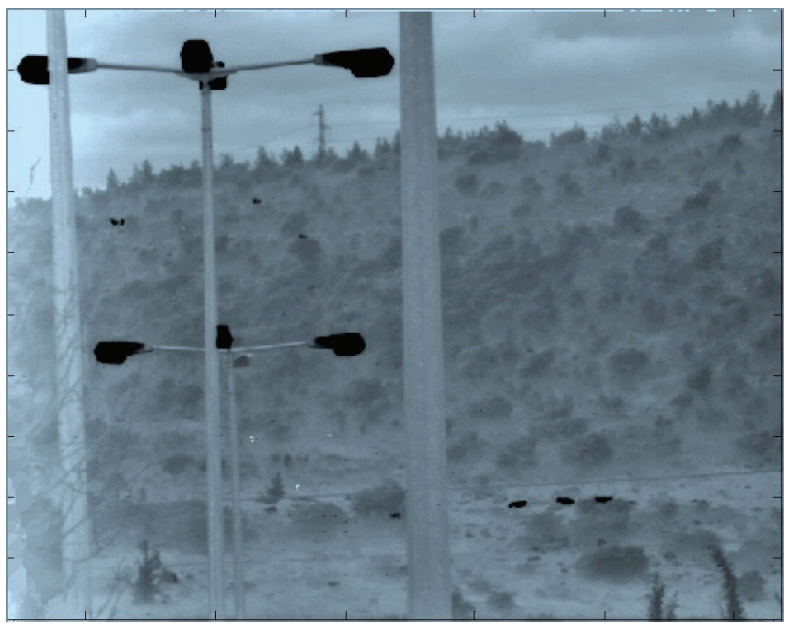

Fig. 17 Image taken at $150 \mathrm{~K}$ with an $\mathrm{nBn} 640 \times 512$ array showing an NE $\Delta \mathrm{T}$ of $20 \mathrm{mK}$. The dark spots on the hill are cows. 


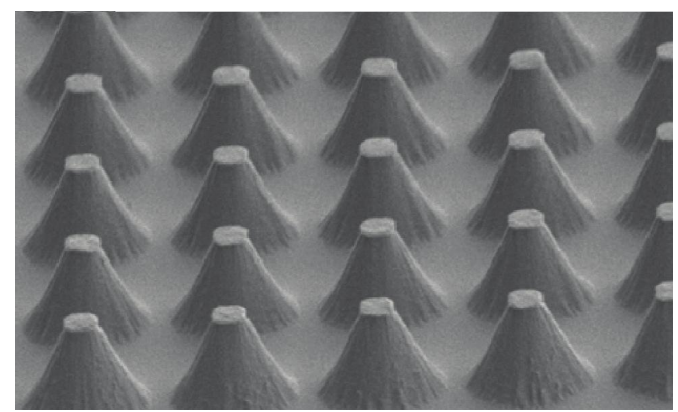

Fig. 18 Pyramidal absorbers on an InAsSb layer were structured to increase photon absorption.

tential advantage if the operating temperature is below the cross-over between diffusion- and generationrecombination-limited regimes. That cross-over point may vary from one material system to another, and within each material system as well, dependent upon the material quality and purity, minority-carrier lifetime, control of doping and bandgap gradients, etc.

The winners in this category will need to be sorted out by investment, time, and experiments. The answer may also depend upon the specific details of the application. Lattice matching to standard substrates may preclude barrier detectors from some applications if the wavelength response is limited to the shorter region of the MWIR band. Novel ideas including pyramid absorbers - see Fig. 18 - to enhance absorption in thin layers was discussed. The detector community has less experience with technologies that have prospered in the laser field, such as cascade quantum structures. We shall see in future years how the current results improve over time.

HOT results for traditional detectors $-\mathrm{HgCdTe}$ and $\mathrm{InSb}$-were presented. Fig. 19 illustrates how NE $\Delta \mathrm{T}$ increases for a $\mathrm{HgCdTe}$ detector with increasing temperature for two spectral MWIR bands. It was noted

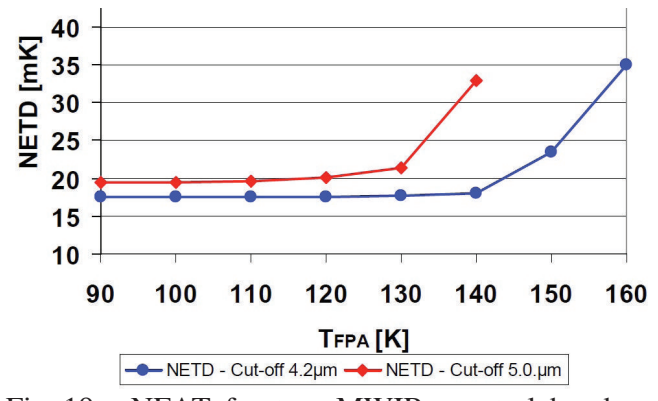

Fig. 19 NEDT for two MWIR spectral bandsnoted - as a function of operating temperature.

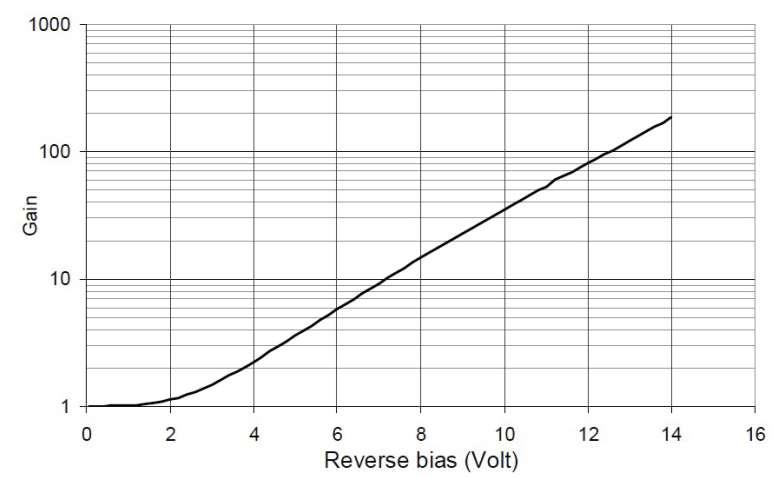

Fig. 20 Gain as a function of bias for a HgCdTe e-APD having a spectral cutoff of $3.1 \mu \mathrm{m}$ at $200 \mathrm{~K}$.

that small-pixel arrays can generally be cooled more quickly and will operate with lower power than large-pixel arrays in the same format. Additional HOT data is part of two papers in the $\mathrm{HgCdTe}$ detector session.

Active imaging technology presentations covered three materials capabable of producing avalanche photodiode (APD) devices:

- InSb

- InGaAs

- $\mathrm{HgCdTe}$

- hole-avalanche devices

- electron-avalanche devices

The electron avalanche gain of a $\mathrm{HgCdTe}$ detector having a $3.1 \mu \mathrm{m}$ cutoff as a function of bias is shown in Figure 20. The gain exceeds 100 at a bias of $12 \mathrm{~V}$. Here the use of shorter cutoff material, compared with earlier results means that such an electron-avalanche detector would need less cooling than one with a cutoff in the range of 4-5 $\mu \mathrm{m}$. On the other hand, the longer cutoff material is better able to provide passive

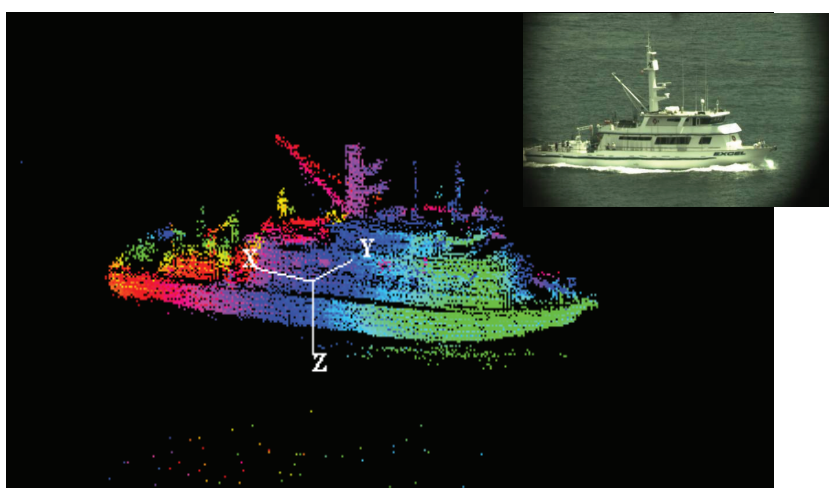

Fig. 21 3D image of a ship (visible insert) made with a scanned linear array of hole-avalanche $\mathrm{HgCdTe}$ detectors. 
thermal imaging in addition to APD operation.

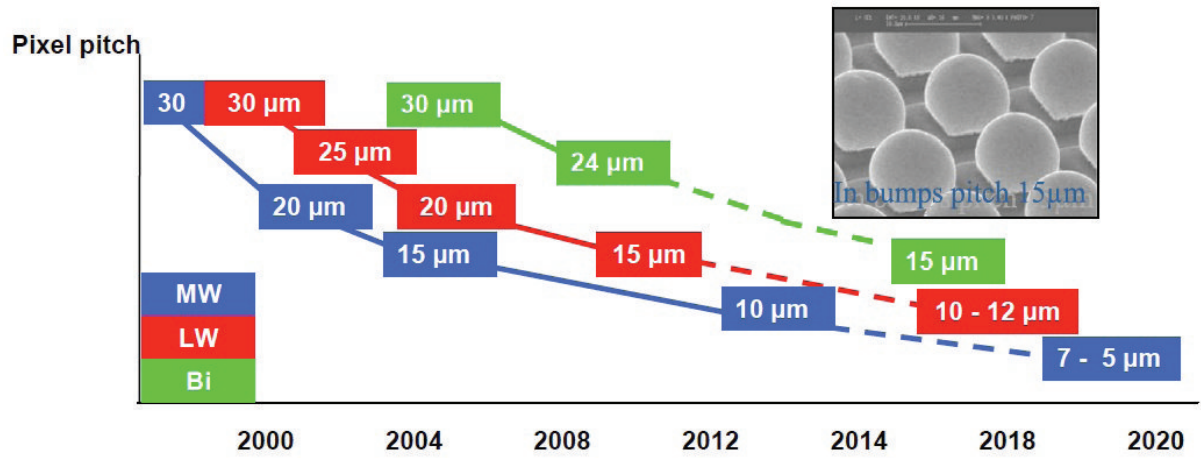

Figure 21 shows the 3D image of a ship taken with a linear array of $\mathrm{HgCdTe}$ holeavalanche APDs in a $256 \times 4$ scanned sensor. These APDs operate up to $1.5 \mathrm{GHz}$ in a linear mode with no afterpulsing effects providing subnanosecond time resolution.

Fig. 24 Progress (solid line) and projections (dashed line) for reducing pixel size for MWIR (blue), LWIR (red), and dual-band FPA applications.

The sensor has a TE cooler for temperature stabilization. Both InGaAs and holeavalanche $\mathrm{HgCdTe}$ can operate at room temperature in the eye-safe SWIR range of 1.5-1.7 $\mu \mathrm{m}$, but cannot provide thermal imagery as well as APD operation.

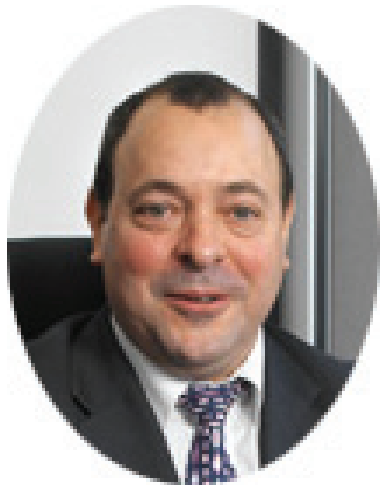

The session on $\mathrm{HgCdTe}$ detectors began with a memorial for colleague Phillippe Tribolet-Figure 22 -who tragically died in the fall of 2010 , much too young. He will be missed by our infrared community.

Fig. 22 Phillippe Tribolet

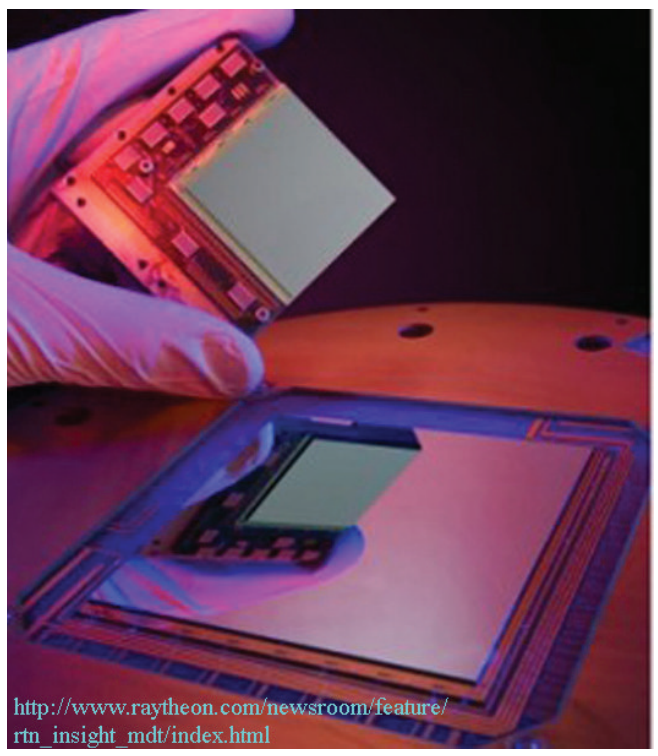

Fig. 23 Image of a $2 \mathrm{~K} \times 2 \mathrm{~K}$ reflected in a $4 \mathrm{~K} \times 4 \mathrm{~K}$ MWIR HgCdTe array grown on silicon.
Advances in $\mathrm{HgCdTe}$ detectors were reported in Session 19. Very large format SWIR and MWIR arrays built with $\mathrm{HgCdTe}$ grown on 6-inch silicon substrates were described $-4 \mathrm{~K} \times 4 \mathrm{~K}$-with $20 \mu \mathrm{m}$ pixels - see Fig. 23. Even larger formats $-8 \mathrm{~K} \times 8 \mathrm{~K}$ - are expected in the near future. $\mathrm{HgCdTe}$ grown on silicon wafers has made this possible, but currently not for LWIR material which is more sensitive to the lattice mismatch.

Progress and projections for reducing pixel size were presented-as shown in Fig. 24 above. This has led to large format MWIR FPAs with $15 \mu \mathrm{m}$ pixels in a 1280 x 1024 tactical format. Excellent imagery results for single and dual MWIR bands were shown - the dualband in a $640 \times 512$ format with $24 \mu \mathrm{m}$ pixels and 20 and $25 \mathrm{mK}$ NE $\Delta \mathrm{T}$, respectively - see Fig. 25.

The combination of GaAs substrates and MOVPE growth has also produced high quality MW/LW results in a $640 \times 512$ format with $20 \mu \mathrm{m}$ pixels. Figure 26 illustrates the NE $\Delta \mathrm{T}$ results for one of these FPAs. In Orlando, Selex demonstrated an extension of this technology to a larger format $-860 \times 480$ with the same pixel pitch and NE $\Delta \mathrm{T}$ performance for $3^{\text {rd }} \mathrm{Gen}$

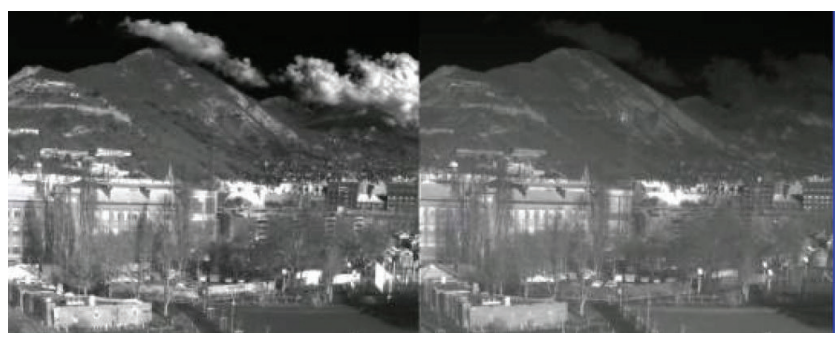

Fig. 25 Imagery taken with a dual-band MW/MW array of $\mathrm{HgCdTe}$ on CdZnTe having $24 \mu \mathrm{m}$ pitch in a $640 \times 512$ format. 


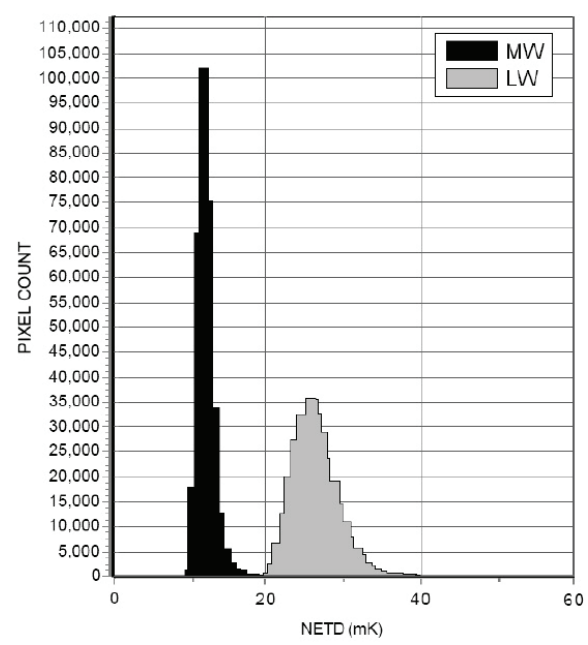

Fig. 26 Histograms of MW and LW band NE $\Delta$ T measured on a HgCdTe-on-GaAs array with $20 \mu$ m pixels.

imaging applications. The HgCdTe-on-GaAs technology also reported progress in MWIR HOT detectors, with impressive imagery shown in Fig. 27 at $160 \mathrm{~K}$ using a $640 \times 512$ format with $16 \mu \mathrm{m}$ pixels. The NE $\Delta \mathrm{T}$ under these conditions was less than $18 \mathrm{mK}$.

Plans were outlined for dual-band 3rd Gen MW/LW technology development also using $\mathrm{HgCdTe} n-p-n$ diodes. Descriptions of a variant of the usual n-p-n structure were presented in two papers-see Fig. 28 for one example. Another novel description was that of a dual-band FPA with a fish-eye lens system partially integrated into the dewar.

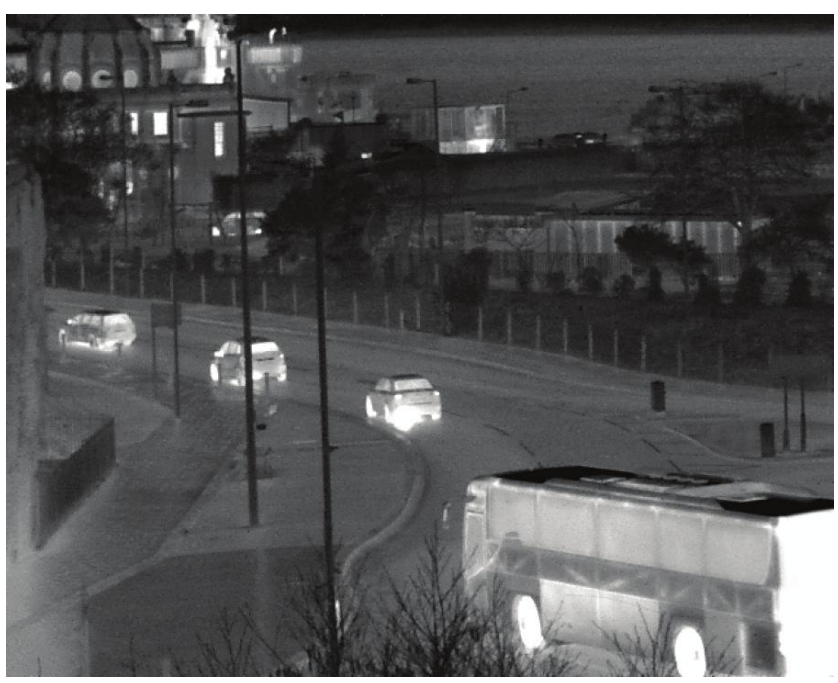

Fig. 27 Image taken at $160 \mathrm{~K}$ with a $\mathrm{HgCdTe}-$ on-GaAs FPA with $16 \mu \mathrm{m}$ pixels.

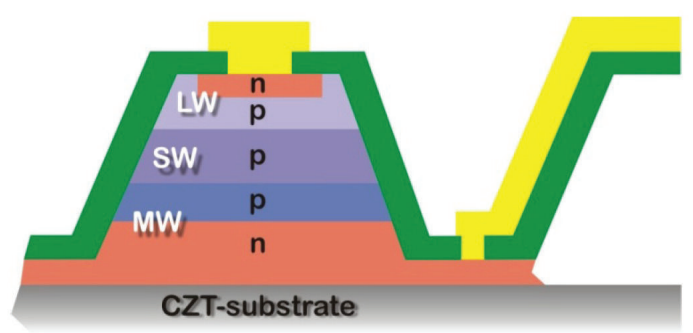

Fig. 28 Doping structure of a novel n-p-n dual-band $\mathrm{HgCdTe}$ pixel structure.

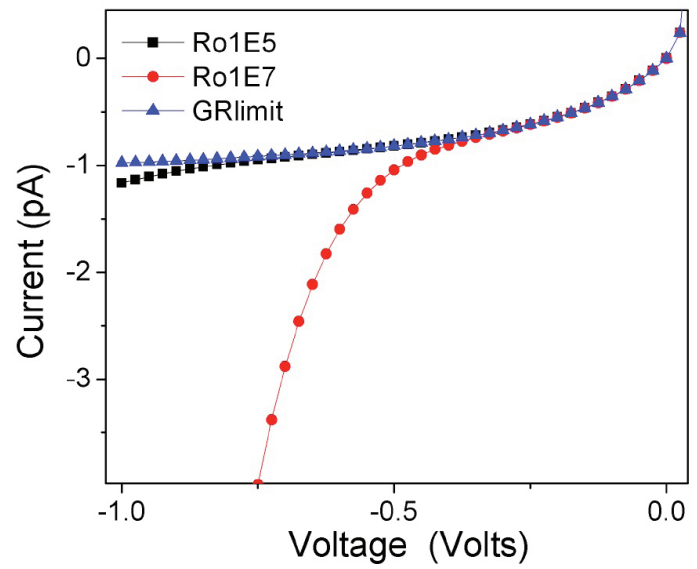

Fig. 29 Illustration of the impact of dislocation density on the reverse-bias characteristics of a $\mathrm{HgCdTe}$ diode.

Elevated dislocation densities are typically encountered when growing $\mathrm{HgCdTe}$ on alternative substrates such as Si or GaAs. A theory was presented to explain this effect. An example of this calculation on the I-V characteristics is shown in Fig. 29.

\section{Cryocoolers}

Session 15 covered cryocoolers. A great effort has been made to miniaturize the detector cryocoolers while retaining or improving the Mean Time To Failure-MTTF. A MicroCryogenic Cooler, MCC, of the Joule-Thomson type was demonstrated for temperatures down to $200 \mathrm{~K}$. MCCs using mixed refrigerants are very promising because they can require only a tenth of the input power of a corresponding TE cooler, and fill a tenth of the volume of a corresponding Stirling cooler, as illustrated in-Fig. 30.

While looking into the future, the MCC presenters envisaged "invisible" cryocoolers which may revolutionize future IR imaging systems. Potential additional size reductions of more than 10 times may be obtained by use of MEMS compressors. 


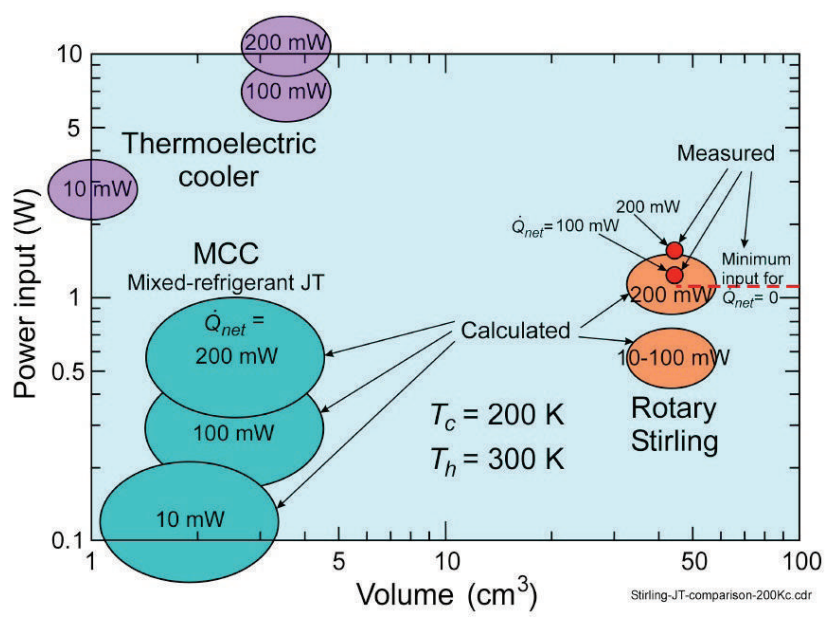

Fig. 30 MCC compared with thermoelectric and Stirling coolers for temperatures down to $200 \mathrm{~K}$.

A novel miniature cryocooler was demonstrated. The reduction of its dimensions was achieved by using moving magnet technology and omitting flexure suspension in split Stirling linear coolers.

Recent trends in developing mini- and micro-satellites for relatively inexpensive missions have prompted attempts to adapt leading-edge tactical cryogenic coolers for suitability in the space environment. The primary emphasis has been on reducing cost, weight and size. Practical and theoretical aspects of adaptation of a microminiature tactical split Stirling cryogenic cooler to the space application were described. The operation of the cooler may give rise to vibrations which will degrade the IR imager's functionality. The authors outlined, in a companion paper, their techniques for reducing these vibrations.
Several coolers of the rotary type were presented. Among these were a sub-micro cooler with fast cooldown and low jitter, one that is particularly suited for intermediate temperatures of 90 to $150 \mathrm{~K}$., and one with a redesigned bearing resulting in doubling of the MTTF.

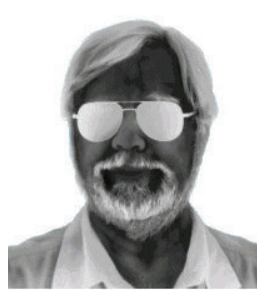

Paul R. Norton

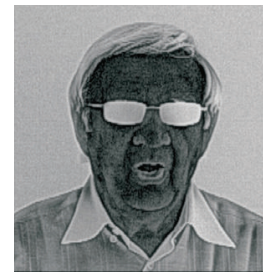

Bjørn F. Andresen

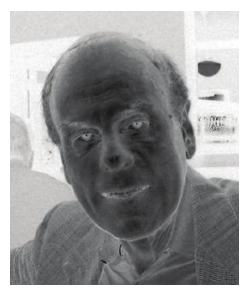

Gabor F. Fulop 
Downloaded From: https://www.spiedigitallibrary.org/conference-proceedings-of-spie on 26 Apr 2023

Terms of Use: https://www.spiedigitallibrary.org/terms-of-use 